\title{
Partial SUSY breaking for asymmetric Gepner models and non-geometric flux vacua
}

\author{
Ralph Blumenhagen, ${ }^{a}$ Michael Fuchs ${ }^{a}$ and Erik Plauschinn ${ }^{b}$ \\ ${ }^{a}$ Max-Planck-Institut für Physik (Werner-Heisenberg-Institut), \\ Föhringer Ring 6, 80805 München, Germany \\ ${ }^{b}$ Arnold Sommerfeld Center for Theoretical Physics, \\ LMU, Theresienstr. 37, 80333 München, Germany \\ E-mail: blumenha@mpp.mpg.de, mfuchs@mpp.mpg.de, erik.plauschinn@lmu.de
}

ABSTRACT: Using the method of simple current extensions, asymmetric Gepner models of Type IIB with $\mathcal{N}=1$ space-time supersymmetry are constructed. The combinatorics of the massless vector fields suggests that these classical Minkowski string vacua provide fully backreacted solutions corresponding to $\mathcal{N}=1$ minima of $\mathcal{N}=2$ gauged supergravity. The latter contain abelian gaugings along the axionic isometries in the hypermultiplet moduli space, and can be considered as Type IIB flux compactifications on Calabi-Yau manifolds equipped with (non-)geometric fluxes. For a particular class of asymmetric Gepner models, we are able to explicitly specify the underlying CICYs and to check necessary conditions for a GSUGRA interpretation. If this conjecture is correct, there exists a large class of exactly solvable non-geometric flux compactifications on CY threefolds.

Keywords: Conformal Field Theory, Supersymmetry Breaking, Flux compactifications

ARXIV EPRINT: 1608.00595 


\section{Contents}

1 Introduction 1

2 SUSY breaking in Gepner models 3

2.1 Review of Gepner construction 3

2.2 Simple current extension 5

2.3 Asymmetric Gepner models 6

$3 \quad$ Partial SUSY-breaking in $\mathcal{N}=2$ GSUGRA $\quad 8$

3.1 Basics of $\mathcal{N}=2$ GSUGRA 8

$\begin{array}{ll}3.2 \text { Gaugings via background fluxes } & 11\end{array}$

$\begin{array}{lll}3.3 & \text { Partial supersymmetry breaking } & 12\end{array}$

4 ACFT-GSUGRA correspondence $\quad \mathbf{1 5}$

4.1 Procedure 16

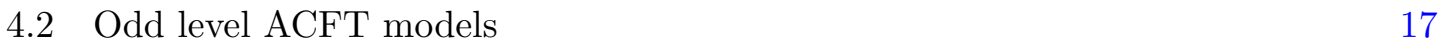

4.3 Level six ACFT model 20

4.4 Level ten ACFT model 22

4.5 Check of GSUGRA constraints 23

5 Conclusions 23

$\begin{array}{ll}\text { A Hodge numbers for CICY } & 25\end{array}$

\section{Introduction}

Classical vacua of string theory are described by two-dimensional conformal field theories (CFTs). Space-time supersymmetry is implemented by the spectral flow of the $N=2$ superconformal field theory (SCFT) on the world-sheet, and all perturbative and nonperturbative $\alpha^{\prime}$-corrections are incorporated in the CFT, whereas string-loop and nonperturbative string corrections in the form of D-brane instantons have to be considered in addition. Moreover, it is believed that CFT backgrounds incorporate Neveu-SchwarzNeveu-Schwarz (NS-NS) fluxes but no Ramond-Ramond (R-R) fluxes.

These basic paradigms of string theory define the ideal approach for the construction of (classical) solutions. However, for studying string vacua one usually starts with an effective supergravity theory at lowest order (in $\alpha^{\prime}$ ) and looks for solutions, either arguing or hoping that they extend to the full string equations of motion. In fact, the set of such leading-order solutions and the set of exactly solvable SCFTs are fairly disjoint. Only a few classes are known where agreement has been achieved, mostly by indirect arguments. 
The best controlled class is build upon toroidal compactifications and orbifolds thereof, which are related to (quotients of) free conformal field theories. Another class is given by Gepner models [1,2]. This is a construction of $N=2$ SCFTs that are argued to provide exact solutions to a string propagating on a Calabi-Yau (CY) manifold at a very specific point in its moduli space. This point is deep inside the Kähler moduli space, where sizes are of the order of the string-scale $\sqrt{\alpha^{\prime}}$. In view of the fact that the Ricci-flat metric on the $\mathrm{CY}$ and therefore the corresponding non-linear sigma model cannot even be written down, this is still quite a remarkable result. This correspondence has been argued for by comparison of massless spectra, the chiral ring and discrete symmetries.

Using the method of simple current extensions of partition functions [3, 4], this construction was generalized to $(0,2)$ heterotic SCFT models in [5], which were argued to correspond to monad bundles with structure group $\mathrm{SU}(n)$ on complete intersection CalabiYau (CICY) manifolds [6, 7]. However, for most of the solutions of the leading-order SUGRA equations of motion the exact CFT is not known. Moreover, there exist large classes of exactly known SCFTs, which so far did not found an interpretation in terms supergravity solutions. Among these are e.g. the in general asymmetric simple current extensions of Gepner models reported in [8] (see also $[9,10]$ ).

A large class of vacua, the so-called string landscape, arises in flux compactifications of string theory. Starting with for instance a Calabi-Yau manifold, some of the moduli can be stabilized by such fluxes, which is important for applications to string phenomenology and string cosmology. Turning on fluxes leads to an $\mathcal{N}=2$ gauged supergravity (GSUGRA) theory whose vacuum structure can be quite involved. In particular, the most generic gaugings include so-called non-geometric fluxes. Such gaugings in $\mathcal{N}=2$ GSUGRA can be described in the framework of $\mathrm{SU}(3) \times \mathrm{SU}(3)$ structures [11-15]. These can also be described in double field theory (for reviews see [16-18]) and their understanding has been under investigation during recent years [19]. For instance, starting with a Type II compactification on a Calabi-Yau threefold, turning on generic NS-NS and R-R fluxes leads to an $\mathcal{N}=2$ gauged supergravity theory, where abelian isometries along the axionic directions in the hypermultiplet moduli space are gauged [12, 20-24].

For a long time it was not clear whether this GSUGRA theory admits $\mathcal{N}=1$ minima, i.e. whether partial supersymmetry breaking from $\mathcal{N}=2$ to $\mathcal{N}=1$ is possible. The first example was constructed in [25], and in a more general context this issue was resolved in the series of papers [26-29]. The main result is that for partially-broken Minkowski vacua one needs at least two gauged isometries through both electric and magnetic fluxes, with one of them being non-geometric. Of course this is just a solution to the GSUGRA equations of motion and it is not clear whether they uplift to genuine SCFTs. In fact, from a string theory perspective one expects that turning on general types of fluxes leads to a strong backreaction [30]. In particular, without having a dilute flux limit available for non-geometric fluxes, it is not clear whether the ansatz of a CY with constant fluxes can capture the true vacuum structure.

In this paper we consider a subclass of the asymmetric simple current extensions of Gepner models and suggest that these can be identified with the fully backreacted solution of partially SUSY breaking Minkowski vacua of $\mathcal{N}=2$ GSUGRA. We cannot prove this 
conjecture, but we collect some evidence for it. In particular, for a set of such asymmetric CFTs (ACFTs) we give an explicit proposal for the underlying CICY manifold of the $\mathcal{N}=2$ GSUGRA. As in the $(0,2)$ setting $[6,7]$, the guiding principle is the combinatorics of the massless states in the asymmetric Gepner model, which reveals information about the weight of the coordinates of an underlying weighted projective space. If our conjecture is correct, it has interesting consequences:

- Partial supersymmetry breaking is possible in string theory even beyond leading order in $\alpha^{\prime}$.

- Minima of an $\mathcal{N}=2$ GSUGRA theory can correspond to classical minima of string theory.

- Non-geometric fluxes are part of the string degrees of freedom and correspond to ACFTs. This correspondence is also obtained for asymmetric orbifolds of tori [31-34].

Such asymmetric Gepner models [8] have also been considered more recently in the two papers $[35,36]$ so that our approach should be considered as an extension of their work. In the present paper we go beyond them in two aspects. First, we allow more general simple currents and second we do suggest that the ACFT constructions are related to GSUGRA minima with partial SUSY breaking.

This paper is organized as follows: in section 2 we briefly review the construction of Gepner models and their partial breaking to $\mathcal{N}=1$ supersymmetry. In section 3 we summarize partial SUSY breaking from a supergravity point of view, and derive bounds on the spectrum after the breaking. In section 4 we discuss explicit examples for our proposed ACFT-GSUGRA correspondence, and section 5 contains our conclusions.

\section{SUSY breaking in Gepner models}

Since the seminal work of D. Gepner $[1,2]$ it is known that there exists a class of $N=2$ supersymmetric CFTs that describe special points in the moduli space of Calabi-Yau compactifications. These so-called Gepner models are the starting point of our construction. It is also known that the simple current construction can lead to modular invariant partition functions that break e.g. the left-moving space-time supersymmetry. In this way one obtains $(0,2)$ or $(1,2)$ superconformal field theories describing classical $\mathcal{N}=1$ Minkowskitype string vacua. Thus, such special kinds of simple currents provide a CFT realization of partial supersymmetry breaking. In this section, we briefly recall the structure of Gepner models and the simple current construction. For more details we refer the reader to the original literature, and for instance to [37] for a recent review.

\subsection{Review of Gepner construction}

In light-cone gauge, the internal sector of a Type II compactification to four dimensions with $\mathcal{N}=2$ supersymmetry is given by tensor products of the rational models of the $N=2$ super Virasoro algebra with total central charge $c=9$. Space-time supersymmetry 
is achieved by a GSO projection, which can be described by a certain simple current in the superconformal field theory.

The minimal models are parametrized by the level $k=1,2, \ldots$ and have central charge

$$
c=\frac{3 k}{k+2} \text {. }
$$

Since $c<3$, the required value $c=9$ is achieved by using tensor products of such minimal models $\bigotimes_{j=1}^{r}\left(k_{j}\right)$. The finite number of irreducible representations of the $N=2$ Virasoro algebra of each unitary model are labeled by the three integers $(l, m, s)$ in the range

$$
l=0, \ldots k, \quad m=-k-1,-k, \ldots k+2, \quad s=-1,0,1,2,
$$

with $l+m+s=0 \bmod 2$. Actually, the identification between $(l, m, s)$ and $(k-l, m+k+$ $2, s+2)$ reveals that the range (2.2) is a double covering of the allowed representations. The conformal dimension and charge of the highest weight state with label $(l, m, s)$ are

$$
\begin{aligned}
\Delta_{m, s}^{l} & =\frac{l(l+2)-m^{2}}{4(k+2)}+\frac{s^{2}}{8}, \\
q_{m, s}^{l} & =\frac{m}{(k+2)}-\frac{s}{2} .
\end{aligned}
$$

Note that these formulas are only correct modulo one and two, respectively. To obtain the precise conformal dimension $h$ and charge from (2.3) one first shifts the labels into the standard range $|m-s| \leq l$ by using the shift symmetries $m \rightarrow m+2 k+4, s \rightarrow s+4$ and the reflection symmetry. The NS-sector consists of those representations with even $s$, while the ones with odd $s$ are from the R-sector.

In addition to the internal $N=2$ sector, one has the contributions with $c=3$ from the two uncompactified directions. The two world-sheet fermions $\psi^{2,3}$ generate a $\mathrm{U}(1)_{2}=$ $\mathrm{SO}(2)_{1}$ model whose four irreducible representations $(c, o, s, v)$ are labeled by $s_{0}=-1, \ldots, 2$ with highest weight and charge modulo one and two respectively

$$
\Delta_{s_{0}}=\frac{s_{0}^{2}}{8}, \quad q_{s_{0}}=-\frac{s_{0}}{2} .
$$

The GSO projection, guaranteeing absence of tachyons and space-time supersymmetry, means in the Gepner case that one projects onto states with odd overall U(1) charge $Q_{\text {tot }}=q_{s_{0}}+\sum_{j=1}^{r} q_{m_{j}, s_{j}}^{l_{j}}$. Moreover, for having world-sheet supersymmetry with

$$
G_{\mathrm{tot}}=\sum_{j=1}^{r} G_{j}+: \partial_{z} X^{\mu} \psi_{\mu}:
$$

beging the overall $N=1$ world-sheet supercurrent in the product theory, one has to ensure that in the tensor product only states from the NS respectively the R sectors couple among themselves.

These projections are described most conveniently in the following notation. First one defines some multi-labels

$$
\lambda=\left(l_{1}, \ldots, l_{r}\right), \quad \mu=\left(s_{0} ; m_{1}, \ldots m_{r} ; s_{1}, \ldots, s_{r}\right),
$$


and the respective characters

$$
\chi_{\mu}^{\lambda}(q)=\chi_{s_{0}}(q) \chi_{m_{1}, s_{1}}^{l_{1}}(q) \ldots \chi_{m_{r}, s_{r}}^{l_{r}}(q) .
$$

In terms of the vectors

$$
\beta_{0}=(1 ; 1, \ldots, 1 ; 1, \ldots, 1), \quad \beta_{j}=(2 ; 0, \ldots, 0 ; 0, \ldots, 0, \underbrace{2}_{j^{\text {th }}}, 0, \ldots, 0),
$$

and the following product

$$
\begin{aligned}
Q_{\mathrm{tot}} & =2 \beta_{0} \bullet \mu=-\frac{s_{0}}{2}-\sum_{j=1}^{r} \frac{s_{j}}{2}+\sum_{j=1}^{r} \frac{m_{j}}{k_{j}+2}, \\
\beta_{j} \bullet \mu & =-\frac{s_{0}}{2}-\frac{s_{j}}{2},
\end{aligned}
$$

the projections one has to implement are simply $Q_{\text {tot }}=2 \beta_{0} \bullet \mu \in 2 \mathbb{Z}+1$ and $\beta_{j} \bullet \mu \in \mathbb{Z}$ for all $j=1, \ldots r$. Gepner has shown that the following GSO projected partition function

$$
Z_{D}(\tau, \bar{\tau})=\frac{1}{2^{r}} \frac{(\operatorname{Im} \tau)^{-2}}{|\eta(q)|^{4}} \sum_{b_{0}=0}^{K-1} \sum_{b_{1}, \ldots, b_{r}=0}^{1} \sum_{\lambda, \mu}^{\beta}(-1)^{s_{0}} \chi_{\mu}^{\lambda}(q) \chi_{\mu+b_{0} \beta_{0}+b_{1} \beta_{1}+\ldots b_{r} \beta_{r}}^{\lambda}(\bar{q})
$$

is indeed modular invariant and vanishes due to space-time supersymmetry. Here $K=$ $\operatorname{lcm}\left(4,2 k_{j}+4\right)$ and $\sum^{\beta}$ means that the sum is restricted to those $\lambda$ and $\mu$ in the range (2.2) satisfying $2 \beta_{0} \bullet \mu \in 2 \mathbb{Z}+1$ and $\beta_{j} \bullet \mu \in \mathbb{Z}$.

\subsection{Simple current extension}

Recall that for a given conformal field theory there exists a very general way to construct modular invariant partition functions via an extension of the chiral symmetry algebra by some element of the set of simple currents $[3,4]$. These simple currents are primary fields $J_{a}$ whose operator product expansion with any other primary field $\phi_{i}$ only involves one particular other primary field, i.e.

$$
J_{a} \times \phi_{i}=\phi_{J(i)}
$$

under fusion. Due to the associativity of the fusion rules it follows that the OPE of two simple currents yields again a simple current, so that in a rational CFT the set of simple currents forms a finite abelian group $\mathcal{S}$ under the fusion product. Being finite there must exist a length $\mathcal{N}_{a}$ where $\mathcal{J}_{a}^{\mathcal{N}_{a}}=1$. The set $\left\{J_{a}, J_{a}^{2}, \ldots, J_{a}^{\mathcal{N}_{a}}\right\}$ forms an abelian subgroup of $\mathcal{S}$ isomorphic to $\mathbb{Z}_{\mathcal{N}_{a}}$ with $\left(J_{a}^{n}\right)^{C} \equiv\left(J_{a}^{n}\right)^{-1}=J_{a}^{\mathcal{N}_{a}-n}$. Similarly, every simple current groups the primary fields into orbits $\left\{\phi_{i}, J_{a} \times \phi_{i}, J_{a}^{2} \times \phi_{i}, \ldots, J_{a}^{\mathcal{N}_{a}^{i}-1} \times \phi_{i}\right\}$ whose length $\mathcal{N}_{a}^{i}$ is a divisor of $\mathcal{N}_{a}$.

The crucial observation is that the action of simple currents in a RCFT implies the existence of a conserved quantity for every primary $\phi_{i}$, the monodromy charge $Q_{i}^{(a)}$, defined by

$$
J_{a}(z) \phi_{i}(w)=(z-w)^{-Q_{i}^{(a)}} \phi_{J(i)}(w)+\ldots
$$


The monodromy of the identity being 1 , it is clear that $Q_{i}^{(a)}=\frac{t_{a}^{i}}{\mathcal{N}_{a}} \bmod 1$ for some integer $t_{a}^{i}$. On the other hand, the monodromy is given by the conformal dimensions of the primary and the simple current as

$$
Q_{i}^{(a)}=h\left(\phi_{i}\right)+h\left(J_{a}\right)-h\left(J_{a} \times \phi_{i}\right) \bmod 1,
$$

from which one can derive that

$$
Q_{i}^{(a)}\left(J_{a}^{n} \times \phi_{i}\right)=\frac{t_{a}^{i}+r_{a} n}{\mathcal{N}_{a}} \bmod 1 .
$$

Here the monodromy parameter $r_{a}$ is defined such that

$$
h\left(J_{a}\right)=\frac{r_{a}\left(\mathcal{N}_{a}-1\right)}{2 \mathcal{N}_{a}} \bmod 1 .
$$

One can prove that a simple current $J_{a}$ with even monodromy parameter $r_{a}$ induces the modular invariant partition function

$$
Z_{a}(\tau, \bar{\tau})=\vec{\chi}^{T}(\tau) M\left(J_{a}\right) \vec{\chi}(\bar{\tau})=\sum_{k, l} \chi_{k}(\tau)\left(M_{a}\right)_{k l} \chi_{l}(\bar{\tau})
$$

where

$$
\left(M_{a}\right)_{k l}=\sum_{p=1}^{\mathcal{N}_{a}} \delta\left(\phi_{k}, J_{a}^{p} \times \phi_{l}\right) \delta^{(1)}\left(\hat{Q}^{(a)}\left(\phi_{k}\right)+\hat{Q}^{(a)}\left(\phi_{l}\right)\right)
$$

and

$$
\hat{Q}^{(a)}\left(\phi_{i}\right)=\frac{t_{a}^{i}}{2 \mathcal{N}_{a}} \bmod 1
$$

Note that the proof relies on the fact that $r_{a}$ is even, which can always be arranged for odd $\mathcal{N}_{a}$, with $r_{a}$ being defined only $\bmod \mathcal{N}_{a}$.

Given two modular invariant matrices $M_{a_{1}}$ and $M_{a_{2}}$, it is clear that $Z_{a_{1}, a_{2}}=$ $\frac{1}{N} \sum_{k, l, m} \chi_{l}\left(M_{a_{1}}\right)_{l k}\left(M_{a_{2}}\right)_{k m} \chi_{m}$ is another modular invariant partition function with obvious generalizations for several $M_{a_{i}}$; the normalization factor $N$ ensures that the vacuum appears precisely once in $Z_{a_{1}, a_{2}}$. The matrices $M$ are also seen to commute if the respective simple currents $J_{a_{1}}$ and $J_{a_{2}}$ are mutually local, i.e. if their relative monodromy charge $Q^{\left(a_{1}\right)}\left(J_{a_{2}}\right)=0 \bmod 1$.

\subsection{Asymmetric Gepner models}

In order to directly apply the simple current extension to the Gepner model, one needs to apply the bosonic string map that exchanges $\mathrm{SO}(2)_{1} \rightarrow \mathrm{SO}(10)_{1} \otimes\left(E_{8}\right)_{1}$ and maps the four representations as

$$
\phi_{\mathrm{bsm}}:(o, v, s, c) \rightarrow(v, o,-c,-s) \otimes 1 .
$$

In this way one obtains a purely bosonic CFT without any minus signs in the modular invariant partition function. Given the fusion rules

$$
\phi_{\left(m_{1}, s_{1}\right)}^{0} \times \phi_{\left(m_{2}, s_{2}\right)}^{l_{2}}=\phi_{\left(m_{1}+m_{2}, s_{1}+s_{2}\right)}^{l_{2}},
$$


we conclude that the simple currents $J$ of the Gepner model under consideration can be labeled by the vector

$$
J=\left(0 m_{1} s_{1}\right) \ldots\left(0 m_{5} s_{5}\right)\left(s_{0}\right) .
$$

The Gepner partition function can then be expressed as the simple current extension

$$
\left.Z_{\mathrm{Gepner}}(\tau, \bar{\tau}) \sim \vec{\chi}^{T}(\tau) M\left(J_{\mathrm{GSO}}\right) \prod_{r=1}^{5} M\left(J_{i}\right) \vec{\chi}(\bar{\tau})\right|_{\phi_{\mathrm{bsm}}^{-1}}
$$

with the bosonic string map applied backwards at the end. The simple currents are given by

$$
\begin{aligned}
& J_{\mathrm{GSO}}=\left(\begin{array}{lll}
0 & 1 & 1
\end{array}\right) \ldots\left(\begin{array}{lll}
0 & 1 & 1
\end{array}\right)(s), \\
& J_{i}=\left(\begin{array}{lll}
0 & 0 & 0
\end{array}\right) \ldots \underbrace{\left(\begin{array}{lll}
0 & 0 & 2
\end{array}\right)}_{i^{\text {th }}} \ldots\left(\begin{array}{llll}
0 & 0 & 0
\end{array}\right)(v) .
\end{aligned}
$$

The $J_{i}$ are also called alignment simple currents. Having a minimal model with even level e.g. in the last factor one can also use its D-type modular invariant by adding the simple current

$$
J_{D}=\left(\begin{array}{lll}
0 & 0 & 0
\end{array}\right) \ldots\left(\begin{array}{lll}
0 & 0 & 0
\end{array}\right)\left(\begin{array}{lll}
0 & k
\end{array}+22\right)(o)
$$

in the partition function. All these simple currents above are relatively local to each other.

The massless spectrum can be read off from the partition function and consists of the $\mathcal{N}=2$ supergravity multiplet, the universal hypermultiplet, $N_{V}$ vectormultiplets and additional $N_{H}-1$ hypermultiplets. For the Type IIB superstring this CFT describes a special point in the moduli space of a Calabi-Yau compactification with Hodge numbers $h^{2,1}=N_{V}$ and $h^{1,1}=N_{H}-1$. Note that the supergravity multiplet contains the graviphoton and the universal hypermultiplet contains the dilaton and the four-dimensional Kalb-Ramond field $B_{\mu \nu}$.

As exploited in the heterotic context in $[5,8]$ and for Type II in $[35,36]$, the method of simple currents is very well suited to construct asymmetric CFTs. One only has to further extend the Gepner partition function by a simple current $J_{\mathrm{ACFT}}$ that is not local with respect to $J_{\mathrm{GSO}}$ and/or $J_{i}$. Indeed in the partition function

$$
\left.Z_{\mathrm{ACFT}}(\tau, \bar{\tau}) \sim \vec{\chi}^{T}(\tau) M\left(J_{\mathrm{ACFT}}\right) M\left(J_{\mathrm{GSO}}\right) \prod_{r=1}^{5} M\left(J_{i}\right) \vec{\chi}(\bar{\tau})\right|_{\phi_{\mathrm{bsm}}^{-1}}
$$

the spectral flow (left-moving supercharge) does not act on the left-moving side leading to an asymmetric CFT-model with only $\mathcal{N}=1$ space-time supersymmetry.

If $J_{\mathrm{ACFT}}$ does not commute with $J_{\mathrm{GSO}}$ then the left-moving space-time supersymmetry is broken. For interpreting the model as a type II compactification, it was so far assumed $[8,35,36]$ that $J_{\mathrm{ACFT}}$ has to commute with the simple currents $J_{i}$, that were implemented in order to realize $N=1$ supersymmetry on the tensor product with the supercurrent given by (2.5). This means that $J_{\mathrm{ACFT}}$ should contain only NS or R entries for each tensor factor. For our purposes we will not require this strong condition, as from the point of view of the central charge $c=(12,12)$, modular invariance, absence of tachyons 
and the arising multiplet structure of the massless spectrum we do not see any difference. Indeed we think that this mixing of NS and R entries in $J_{\mathrm{ACFT}}$ does not necessarily break the left-moving $N=1$ world-sheet supersymmetry. As we will see in section 4.2 , in this class we can construct models with even $\mathcal{N}=2$ space-time supersymmetry.

In any case, by construction (2.25) is a classical tachyon-free string vacuum with the central charge $(12,12)$ that has vanishing cosmological constant, i.e. it is a Minkowski vacuum. Of course one can add more simple currents, but in this paper we only consider the simplest case with only a single one. The massless spectrum still fits into $\mathcal{N}=1$ supermultiplets. From the vacuum orbit one gets the $\mathcal{N}=1$ supergravity multiplet and a chiral superfield containing the dilaton and another pseudo-scalar. The graviphoton is not massless anymore. From the so-called matter orbits, one gets three kinds of massless states. Left-right combinations of the form

$$
(h=3 / 8)(s) \otimes[(\bar{h}=1 / 2, \bar{q}=1)(o)+(\bar{h}=3 / 8, \bar{q}=-1 / 2)(s)]
$$

lead to massless $\mathcal{N}=1$ vectormultiplets, combinations

$$
(h=3 / 8)(c) \otimes[(\bar{h}=1 / 2, \bar{q}=1)(o)+(\bar{h}=3 / 8, \bar{q}=-1 / 2)(s)]
$$

to massless R-R axion-like chiral multiplets and

$$
(h=1 / 2)(o) \otimes[(\bar{h}=1 / 2, \bar{q}=1)(o)+(\bar{h}=3 / 8, \bar{q}=-1 / 2)(s)]
$$

to NS-NS scalar chiral multiplets. Therefore, the latter three classes of massless states are described by three numbers $\left(N_{V}, N_{\text {ax }} ; N_{0}\right)$.

In section 4 we will discuss a couple of examples for $J_{\mathrm{ACFT}}$. All these are very simple, in the sense that they trivially act on most of the tensor factors. Recall that it is the aim of this paper to provide arguments for the identification of asymmetric Type IIB Gepner models (2.25) with $\mathcal{N}=1$ Minkowski vacua of $\mathcal{N}=2$ gauged supergravity models. Therefore, let us first recollect the structure of $\mathcal{N}=2$ GSUGRA and its partial supersymmetry breaking vacua.

\section{Partial SUSY-breaking in $\mathcal{N}=2$ GSUGRA}

In this section we review some aspects of $\mathcal{N}=2$ gauged supergravity theories and their partial breaking to $\mathcal{N}=1$, for which we mostly follow [27-29]. We consider GSUGRAs resulting from flux compactifications of type IIB superstring theories, which are related to abelian gaugings along the axionic directions in the hypermultiplet moduli space.

\subsection{Basics of $\mathcal{N}=2$ GSUGRA}

We begin by reviewing $\mathcal{N}=2$ gauged supergravity theories in four dimensions, which arise from Calabi-Yau compactifications of superstring theory with fluxes. The field content in the four-dimensional theory is given by one supergravity multiplet, $N_{V}$ vector-multiplets 
and $N_{H}$ hyper-multiplets. For type IIB string theory we have $N_{V}=h^{2,1}$ and $N_{H}=h^{1,1}+1$. The multiplets contain the following degrees of freedom

$$
\begin{array}{ll}
\text { massless } \mathcal{N}=2 \text { gravity } & \mathcal{G}_{(2)}=1 \cdot[2]+2 \cdot\left[\frac{3}{2}\right]+1 \cdot[1]=(2)_{\mathrm{b}}+(4)_{\mathrm{f}}+(2)_{\mathrm{b}}, \\
\text { massless } \mathcal{N}=2 \text { vector } & \mathcal{V}_{(2)}=1 \cdot[1]+2 \cdot\left[\frac{1}{2}\right]+2 \cdot[0]=(2)_{\mathrm{b}}+(4)_{\mathrm{f}}+(2)_{\mathrm{b}}, \\
\text { massless } \mathcal{N}=2 \text { hyper } & \mathcal{H}_{(2)}=2 \cdot\left[\frac{1}{2}\right]+4 \cdot[0] \quad=(4)_{\mathrm{f}}+(4)_{\mathrm{b}},
\end{array}
$$

where the number in a square bracket indicates the spin and the number in parenthesis counts the real bosonic and fermionic degrees of freedom. Compactification of type IIB string theory on Calabi-Yau manifolds are well-understood, and in the following we therefore only summarize the features needed here.

NS-NS sector. One introduces a symplectic basis for the third cohomology group of the Calabi-Yau three-fold $\mathcal{M}$ as $\left\{\alpha_{\Lambda}, \beta^{\Lambda}\right\} \in H^{3}(\mathcal{M})$ with $\Lambda=0, \ldots, h^{2,1}$. The holomorphic three-form $\Omega$ can be expanded as

$$
\Omega=X^{\Lambda} \alpha_{\Lambda}-F_{\Lambda} \beta^{\Lambda}=\left(\alpha_{\Lambda}, \beta^{\Lambda}\right) \cdot V_{2},
$$

where we follow the conventions in $[19,24]$ and introduced a $\left(2 h^{2,1}+2\right)$-dimensional vector as $V_{2}^{T}=\left(X^{\Lambda},-F_{\Lambda}\right)$. The periods $X^{\Lambda}$ are projective coordinates on the moduli space, and are related to the complex-structure moduli through $z^{a}=X^{a} / X^{0}$ where $a=1, \ldots, h^{2,1}$. We also mention that it is usually assumed that the periods $F_{\Lambda}$ can be written as the derivative of a prepotential $F(X)$ with respect to $X^{\Lambda}$, that is $F_{\Lambda}=\partial F / \partial X^{\Lambda}$. For later reference we furthermore define the invertible and positive-definite matrix $\mathcal{M}_{1}$, which can be expressed in terms of the period matrix $\mathcal{N}$ as

$$
\mathcal{M}_{1}=\left(\begin{array}{cc}
\mathbb{1} & \operatorname{Re} \mathcal{N} \\
0 & \mathbb{1}
\end{array}\right)\left(\begin{array}{cc}
-\operatorname{Im} \mathcal{N} & 0 \\
0 & -\operatorname{Im} \mathcal{N}^{-1}
\end{array}\right)\left(\begin{array}{cc}
\mathbb{1} & 0 \\
\operatorname{Re} \mathcal{N} & \mathbb{1}
\end{array}\right) .
$$

For the even cohomology of the Calabi-Yau manifold $\mathcal{M}$ one finds a similar special geometry. We introduce bases of the form $\left\{\omega_{\mathrm{A}}\right\} \in H^{1,1}(\mathcal{M})$ and $\left\{\sigma^{\mathrm{A}}\right\} \in H^{2,2}(\mathcal{M})$ with $\mathrm{A}=1, \ldots, h^{1,1}$. We can group these two- and four-forms together with the zero- and sixform as $\left\{\omega_{A}\right\}=\left\{1, \omega_{\mathrm{A}}\right\}$ and $\left\{\sigma^{A}\right\}=\left\{\frac{\sqrt{g}}{\mathcal{V}} d x^{6}, \sigma^{\mathrm{A}}\right\}$ with $A=0, \ldots, h^{1,1}$. Here $\mathcal{V}$ is the volume of $\mathcal{M}$. The Kähler form $J$ of $\mathcal{M}$ and the Kalb-Ramond field $B$ are expanded in the basis $\left\{\omega_{\mathrm{A}}\right\}$ in the following way

$$
J=t^{\mathrm{A}} \omega_{\mathrm{A}}, \quad B=b^{\mathrm{A}} \omega_{\mathrm{A}},
$$

and can be combined into a complex field as $\mathcal{J}=B+i J=\mathcal{J}^{\mathrm{A}} \omega_{\mathrm{A}}$. Note that $\mathcal{J}^{A}$ are the $h^{1,1}$ complexified Kähler moduli. We introduce a complex $\left(2 h^{1,1}+2\right)$-dimensional vector $V_{1}$ as

$$
e^{B+i J}=e^{\mathcal{J}}=\left(\omega_{A}, \sigma^{A}\right) \cdot V_{1}
$$


where the components of $V_{1}$ read

$$
V_{1}=\left(\begin{array}{c}
1 \\
\mathcal{J}^{\mathrm{A}} \\
\frac{1}{6} \kappa_{\mathrm{ABC}} \mathcal{J}^{\mathrm{A}} \mathcal{J}^{\mathrm{B}} \mathcal{J}^{\mathrm{C}} \\
\frac{1}{2} \kappa_{\mathrm{ABC}} \mathcal{J}^{\mathrm{B}} \mathcal{J}^{\mathrm{C}}
\end{array}\right) .
$$

Finally, in analogy to (3.3) there exists a positive definite and invertible matrix $\mathcal{M}_{2}$. The precise expressions are not important here, but can be found for instance in section 4.1 of [19].

R-R sector. The Ramond-Ramond sector of type IIB provides additional massless modes, that will play the dominant role in our investigation. The four-dimensional scalar part of the R-R potentials is obtained as follows

$$
\left.\mathcal{C}\right|_{\text {scal. }}=\tilde{\xi}_{0}+\xi^{\mathrm{A}} \omega_{\mathrm{A}}+\tilde{\xi}_{\mathrm{A}} \sigma^{\mathrm{A}}+\xi^{0} \omega_{0}=\left(\omega_{A}, \sigma^{A}\right) \cdot \Xi, \quad \Xi=\left(\begin{array}{c}
\xi^{A} \\
\tilde{\xi}_{A}
\end{array}\right)
$$

where $\mathcal{C}=C_{0}+C_{2}+C_{4}+C_{6}+C_{8}$ is a formal sum of R-R forms in type IIB. This expansion defines a $\left(2 h^{1,1}+2\right)$-dimensional vector $\Xi$ of R-R axions. The pairs $\left(\xi^{\mathrm{A}}, \tilde{\xi}_{\mathrm{A}}\right)$ form $h^{1,1}$ complex axionic scalars, which pair up with the complexified Kähler moduli $\mathcal{J}^{\mathrm{A}}$ to form $h^{1,1}$ hyper-multiplets. The two remaining R-R axions $\left(\xi^{0}, \tilde{\xi}_{0}\right)$ combine with the dilaton $\phi$ and the NS-NS axion $\tilde{\phi}$ to the so-called universal hypermultiplet. The axion $\tilde{\phi}$ is the dual to the four-dimensional Kalb-Ramond field $B_{\mu \nu}$.

Turning now to the four-dimensional vector fields, we expand

$$
C_{4}=A^{\Lambda} \alpha_{\Lambda}+\tilde{A}_{\Lambda} \beta^{\Lambda},
$$

in which $(A, \tilde{A})$ are four-dimensional electric and magnetic vector fields. Eventually, half of these have to be eliminated due to the self-duality condition on the R-R five form, leaving only $h^{2,1}+1$ vectors. But for now we will keep them as separate degrees of freedom. Here the vector field $A^{0}$ is the graviphoton residing in the $\mathcal{N}=2$ supergravity multiplet and the remaining $h^{2,1}$ gauge fields combine with the complex structure moduli to fill out $h^{2,1}$ vector-multiplets. Thus, the bosonic components of the $\mathcal{N}=2$ supergravity multiplets are

$$
\begin{array}{lll}
\text { massless } \mathcal{N}=2 \text { gravity } & \mathcal{G}_{(2)} & \supset\left(g_{\mu \nu}, A^{0}\right), \\
\text { massless } \mathcal{N}=2 \text { vector } & \mathcal{V}_{(2)} \supset\left(A^{a}, z^{a}\right), \\
\text { massless } \mathcal{N}=2 \text { hyper } & \mathcal{H}_{(2)} \supset\left(\mathcal{J}^{\mathrm{A}}, \xi^{\mathrm{A}}, \tilde{\xi}_{\mathrm{A}}\right), \\
& \mathcal{H}_{(2)}^{\text {univ. }} & \supset\left(\phi, \tilde{\phi}, \xi^{0}, \tilde{\xi}_{0}\right) .
\end{array}
$$

The complex structure moduli $z^{a}$ are coordinates on a special Kähler manifold. The $4\left(h^{1,1}+\right.$ 1) scalars in the hypermultiplets form a special hyper-Kähler manifold which is a fibration of dimension $2 h^{1,1}+4$ over a special Kähler manifold described by the $h^{1,1}$ complex Kähler moduli $\mathcal{J}^{\mathrm{A}}$. 


\subsection{Gaugings via background fluxes}

We now turn to compactifications on Calabi-Yau three-folds with background fluxes, which lead to gaugings of (abelian) hyper-multiplet isometries [12, 20-24]. The fluxes can be geometric in the NS-NS sector ( $H$-flux and geometric flux) or R-R sector $\left(F^{(3)}\right.$-flux), or non-geometric in the NS-NS sector $(Q$ - and $R$-flux).

Concerning the R-R sector of type IIB string theory, the three-form flux $F^{(3)}$, can be expanded as

$$
F^{(3)}=-\tilde{\mathrm{F}}^{\Lambda} \alpha_{\Lambda}+\mathrm{F}_{\Lambda} \beta^{\Lambda}=\left(\alpha_{\Lambda} \beta^{\Lambda}\right) \cdot \mathrm{F}^{(3)}, \quad \mathrm{F}^{(3)}=\left(\begin{array}{r}
-\tilde{\mathrm{F}}^{\Lambda} \\
\mathrm{F}_{\Lambda}
\end{array}\right) .
$$

The geometric and non-geometric NS-NS fluxes are conveniently organized into a $\left(2 h^{2,1}+\right.$ $2) \times\left(2 h^{1,1}+2\right)$ matrix as follows (see e.g. [14] $)^{1}$

$$
\mathcal{O}=\left(\begin{array}{cc}
q_{\Lambda}^{A} & f_{\Lambda A} \\
\tilde{q}^{\Lambda A} & \tilde{f}_{A}^{\Lambda}
\end{array}\right)
$$

Note that the $H$ - and $R$-flux are contained in (3.11) via

$$
\begin{array}{ll}
f_{\Lambda 0}=h_{\Lambda}, & \tilde{f}_{0}^{\Lambda}=\tilde{h}^{\Lambda}, \\
q_{\Lambda}{ }^{0}=r_{\Lambda}, & \tilde{q}^{\Lambda 0}=\tilde{r}^{\Lambda} .
\end{array}
$$

These fluxes lead to a gauging of isometries in the hypermultiplet moduli space. More concretely, the shifts along the $2 h^{1,1}+3$ axionic directions $\left\{\xi^{A}, \tilde{\xi}_{A}, \tilde{\phi}\right\}$ are gauged according to

$$
\delta\left(\begin{array}{c}
A \\
\tilde{A}
\end{array}\right)=d \lambda, \quad \delta \Xi=-\mathcal{O}^{T} \cdot \lambda, \quad \delta \tilde{\phi}=-2 \lambda^{T} \cdot \mathrm{F}^{(3)}-\lambda^{T} \cdot C \cdot \tilde{\mathcal{O}} \cdot \Xi,
$$

where $\lambda$ is a $\left(2 h^{2,1}+2\right)$-dimensional vector parametrizing the gauge transformation, and where we have defined the matrices

$$
\tilde{\mathcal{O}}=C \cdot \mathcal{O} \cdot C^{T}, \quad C=\left(\begin{array}{cc}
0 & +\mathbb{1} \\
-\mathbb{1} & 0
\end{array}\right)
$$

with the dimensions of the square matrix $C$ chosen appropriately. In this notation the quadratic constraints (Bianchi identities) for the fluxes can be expressed as

$$
\tilde{\mathcal{O}}^{T} \cdot \mathcal{O}=0, \quad \mathcal{O} \cdot \tilde{\mathcal{O}}^{T}=0
$$

Through such a gauging $n=\operatorname{rank}(\mathcal{O})+\Delta$ gauge fields become massive via the Stückelberg mechanism by eating some of the axions. The extra contribution $\Delta \in\{0,1\}$ is equal to one if the NS-NS axion $\tilde{\phi}$ is gauged as well. (For details on the gauging of $\tilde{\phi}$ see [20].)

\footnotetext{
${ }^{1}$ As compared to [19], we changed our conventions for the fluxes as $\tilde{\mathcal{O}}_{\text {there }}=-\mathcal{O}_{\text {here }}$.
} 
Moreover, the gaugings induce a scalar potential that in general depends on all types of moduli and is given by [24, 38]

$$
\begin{aligned}
V= & \frac{1}{2}\left(\mathrm{~F}^{T}-\Xi^{T} \cdot \tilde{\mathcal{O}}^{T}\right) \cdot \mathcal{M}_{1} \cdot(\mathrm{F}-\tilde{\mathcal{O}} \cdot \Xi) \\
& +\frac{e^{-2 \phi}}{2} V_{1}^{T} \cdot \tilde{\mathcal{O}}^{T} \cdot \mathcal{M}_{1} \cdot \tilde{\mathcal{O}} \cdot \bar{V}_{1} \\
& +\frac{e^{-2 \phi}}{2} V_{2}^{T} \cdot \mathcal{O} \cdot \mathcal{M}_{2} \cdot \mathcal{O}^{T} \cdot \bar{V}_{2} \\
& -\frac{e^{-2 \phi}}{4 \mathcal{V}} V_{2}^{T} \cdot C \cdot \tilde{\mathcal{O}} \cdot\left(V_{1} \times \bar{V}_{1}^{T}+\bar{V}_{1} \times V_{1}^{T}\right) \cdot \tilde{\mathcal{O}}^{T} \cdot C^{T} \cdot \bar{V}_{2}
\end{aligned}
$$

Note that the R-R axions $\Xi$ only appear in the first term and that the scalars gauged via (3.13) do not appear in the scalar potential (3.16). Indeed, due to

$$
\delta_{\lambda}(\tilde{\mathcal{O}} \cdot \Xi)=-\tilde{\mathcal{O}} \cdot \mathcal{O}^{T} \cdot \lambda=0
$$

the scalar potential is gauge invariant. Furthermore, $\tilde{\phi}$ does not appear in (3.16).

To summarize, $n$ of the R-R axions $\Xi$ and the NS-NS axion $\tilde{\phi}$ can become massive via the Stückelberg mechanism, while the remaining axions can still receive a mass from the scalar potential. However, the axions only appear via the combination $\tilde{\mathcal{O}} \cdot \Xi$ which for $h^{2,1}>h^{1,1}$ can be shown to depend only on $h^{11}+1$ combinations of axions. Therefore, at most $h^{1,1}+1$ can receive a mass from the potential. We will see in the next section that for supersymmetric minima, this upper bound is actually smaller.

Conceptually, the scalar potential (3.16) can be obtained from a dimensional reduction of double field theory (DFT) on a Calabi-Yau manifold equipped with NS-NS and R-R fluxes, where the latter are treated as small perturbations around the CY geometry [19]. Furthermore, from a supergravity point of view, (3.16) corresponds to $\mathrm{SU}(3) \times \mathrm{SU}(3)$ structure compactifications [11-15]. However, it is not clear whether four-dimensional GSUGRA can be considered as a low-energy effective action (LEEA) for the light modes in a string compactification. First, even DFT itself is rather a truncation of string theory than an LEEA and second, having non-geometric fluxes turned on implies that in general there does not exist a dilute flux limit for which the backreaction of the fluxes on the CY can be argued to be small [30]. It is thus not clear whether minima of the scalar potential of GSUGRA can be truly uplifted to full classical solutions of the string equations of motion.

\subsection{Partial supersymmetry breaking}

We now briefly describe spontaneous supersymmetry breaking from $\mathcal{N}=2$ to $\mathcal{N}=1$ following the work of $[27,28]$ (see also $[26,39]$ ). As has been shown by these authors, such a breaking is possible if magnetic gaugings and non-geometric fluxes are considered.

Our goal in this section is to deduce bounds on the number of massless vector- and R-R chiral multiplets in $\mathcal{N}=1$ Minkowski vacua of $\mathcal{N}=2$ GSUGRA. In the spontaneouslybroken theory the following multiplets are of importance, which we summarize using the 
same notation as in (3.1):

$$
\begin{array}{lll}
\text { massless } \mathcal{N}=1 \text { gravity } & G_{(1)}=1 \cdot[2]+1 \cdot\left[\frac{3}{2}\right] & =(2)_{\mathrm{b}}+(2)_{\mathrm{f}}, \\
\text { massless } \mathcal{N}=1 \text { vector } & V_{(1)}=1 \cdot[1]+1 \cdot\left[\frac{1}{2}\right] & =(2)_{\mathrm{b}}+(2)_{\mathrm{f}}, \\
\text { massless } \mathcal{N}=1 \text { chiral } & C_{(1)}=1 \cdot\left[\frac{1}{2}\right]+2 \cdot[0] & =(2)_{\mathrm{f}}+(2)_{\mathrm{b}}, \\
\text { massive } \mathcal{N}=1 \text { spin } 3 / 2 & \bar{S}_{(1)}=1 \cdot\left[\frac{3}{2}\right]+2 \cdot[1]+1 \cdot\left[\frac{1}{2}\right]=(4)_{\mathrm{f}}+(6)_{\mathrm{b}}+(2)_{\mathrm{f}}, \\
\text { massive } \mathcal{N}=1 \text { vector } & \bar{V}_{(1)}=1 \cdot[1]+2 \cdot\left[\frac{1}{2}\right]+1 \cdot[0]=(3)_{\mathrm{b}}+(4)_{\mathrm{f}}+(1)_{\mathrm{b}}, \\
\text { massive } \mathcal{N}=1 \text { chiral } & \bar{C}_{(1)}=1 \cdot\left[\frac{1}{2}\right]+2 \cdot[0] & =(2)_{\mathrm{f}}+(2)_{\mathrm{b}} .
\end{array}
$$

The breaking mechanism can be separated into two steps. The first step is responsible for the partial supersymmetry breaking, in which one gravitino of the $\mathcal{N}=2$ gravitymultiplet becomes massive while the other stays massless. The latter will be part of the $\mathcal{N}=1$ gravity multiplet $G_{(1)}$, while the former is part of a massive spin-3/2 multiplet $\bar{S}_{(1)}$. Since the broken theory is required to be $\mathcal{N}=1$ supersymmetric, the massive spin$3 / 2$ multiplet has to contain two massive vector fields, which acquire a mass from the Stückelberg mechanism by eating two gauged axions. The axions can - but do not have to - include the NS-NS field $\tilde{\phi}$.

Furthermore, for an $\mathcal{N}=1$ vacuum with vanishing $\mathrm{R}-\mathrm{R}$ flux, axions $\zeta_{1,2}$ are generically fixed by the complex valued relation

$$
\left(\tilde{\xi}_{A}-G_{A B} \xi^{B}\right) D^{A}=0
$$

where $G_{A B}=\partial_{A} \partial_{B} G$ with $G$ denoting the prepotential for the Kähler moduli space. $D^{A}$ is a constant complex valued vector that specifies the $\mathcal{N}=1$ vacuum (see [27, 28] for more details). Since the axions only appear quadratically in the scalar potential (3.16), these two axions will receive a mass. However, one can imagine that for some boundary values in the Kähler moduli space $G_{A B}$ degenerates such that only one axion is fixed. Thus, in the following we only assume that at least one axion is fixed by (3.19). Recalling then that we have $h^{2,1}+1$ gauge fields to begin with and $2\left(h^{1,1}+1\right)+1$ real axions where only one is from the NS-NS sector, after this first step we are generically left with $N_{V}=h^{2,1}-1$ massless vectors and $N_{\mathrm{ax}}^{\text {real }}=2 h^{1,1}+1-k_{1}$ massless real axions. Here $k_{1} \in\{1,2\}$ denotes the number of axions that became massive due to (3.19).

In the second step, there can be additional gaugings which however do not participate in the partial supersymmetry breaking and can therefore be described in an effective $\mathcal{N}=1$ GSUGRA theory. For $n-2$ additional gaugings, $n-2$ vector fields become massive eating $n-2$ axions. In addition one gets an F-term and a D-term potential, where the axions only appear in the D-terms for the broken abelian gauge fields. In an $\mathcal{N}=1$ SUSY 
preserving Minkowski minimum the D-terms have to vanish providing $n-2$ real conditions. ${ }^{2}$ Therefore, at most $n-2$ additional axions can become massive by this mechanism. The remaining axions are free parameters and therefore massless. In summary, we finally get $N_{V}=h^{2,1}-n+1$ massless vectors and $N_{\mathrm{ax}}^{\text {real }}=2 h^{1,1}-n+3-\left(k_{1}+k_{2}\right)$ real massless axions with $0 \leq k_{2} \leq n-2$ denoting the number of axions that became massive due to the $n-2$ D-terms.

Taking into account the maximal number of gaugings, we have the bound $2 \leq n \leq$ $h^{1,1}+1+\Delta$, where the extra term $\Delta$ appears only for a gauging along the NS-NS axion $\tilde{\phi}$. Therefore, for the number of vector multiplets after gauging we can derive the bound

$$
h^{2,1}-h^{1,1}-\Delta \leq N_{V} \leq h^{2,1}-1 .
$$

Moreover, using $n=h^{2,1}-N_{V}+1$, for the number of real massless axions we find

$$
2\left(h^{1,1}-h^{2,1}+N_{V}\right)+1 \leq N_{\mathrm{ax}}^{\text {real }} \leq 2 h^{1,1}-h^{2,1}+N_{V}+1 .
$$

If the $\tilde{\phi}$-field is gauged then the number of complex R-R axions is $N_{\mathrm{ax}}=N_{\mathrm{ax}}^{\mathrm{real}} / 2$, whereas for an ungauged $\tilde{\phi}$ one has $N_{\mathrm{ax}}=\left(N_{\mathrm{ax}}^{\text {real }}-1\right) / 2$. Thus, for the number of complex R-R axions we derive the bounds

$$
N_{V}-N_{\text {ax }} \leq h^{2,1}-h^{1,1}-\Delta
$$

and

$$
N_{V}-2 N_{\text {ax }} \geq h^{2,1}-2 h^{1,1}-\Delta .
$$

These numbers are to be compared to the ACFT results. Moreover, the dilaton always remains massless for $\mathcal{N}=1$ Minkowski vacua. In the asymmetric Gepner models there exist an accompanying massless NS-NS pseudo-scalar. If the $\tilde{\phi}$-field is not gauged this can be the $\tilde{\phi}$-field itself or, as in the gauged case, it can in principle also be a linear combination of the NS-NS pseudo-scalars appearing for the complex structure and complexified Kähler moduli. By the flux also some of the complex structure moduli and complexified Kähler moduli are fixed. Since these appear in the NS-NS sector, the number of unconstrained ones should be compared with the number of scalars $N_{0}$ in the ACFT.

Remarks. This analysis has been performed in the framework of $\mathcal{N}=2$ GSUGRA, which, as argued before, is not a priori a fully established effective field theory governing the dynamics of string theory on fluxed Calabi-Yau three-folds. First, the question arises whether higher order corrections can induce extra mass terms for the axions so that the bounds can be avoided. Since the axions feature a perturbative continuous shift symmetry, its potential is highly constrained. Like for axion-monodromy, perturbatively this shift

${ }^{2}$ In [29], these D-term conditions were given by

$$
\operatorname{Re}\left(\left(s_{\lambda A}-r_{\lambda}^{C} G_{C A}\right)\left(\operatorname{Im} G^{-1}\right)^{A B}\left(\tilde{\xi}_{B}-\bar{G}_{B D} \xi^{D}\right)-t_{\lambda}\right)=0
$$

where $s_{\lambda A}, r_{\lambda}{ }^{A}$ and $t_{\lambda}$ denote the components of the Killing vectors that are gauged. Note that these conditions depend on the axions and the Kähler moduli. 
symmetry can only be broken by fluxes in a controlled way. As has been argued in [40-42] all higher order corrections are expected to be corrections in terms of the tree-level flux induced potential (instead of the axion field itself). Secondly, there can be non-perturbative corrections for the axions. Since axions arise in the R-R sector of string theory, these would be D-brane instantons, i.e. non-perturbative effects in the string coupling constant. These are not captured by the CFT, which only incorporates world-sheet instantons, i.e. non-perturbative effects in $\alpha^{\prime}$. Following these arguments, the tree-level flux induced potential for the R-R axions is expected to correctly capture the dimension of the axionic moduli space.

However, there is a second more serious issue. In the asymmetric Gepner models all massive modes are of order of the string mass. Therefore, there is no mass hierarchy among the Kaluza-Klein scale, the string scale and the mass scale of the massive moduli. It is thus unclear whether a GSUGRA theory for the initially massless modes can reliably describe the full dynamics of mass generation in string theory. Of course gauge symmetry and shiftsymmetry do protect masses to a certain degree, but there can be subtle effects that for instance generate masses for the R-R axions via couplings to massive Kaluza-Klein modes.

It is precisely one of the objectives of this work to investigate to which degree minima of GSUGRA theories do provide or at least hint at true flux vacua of string theory. For that purpose let us now consider concrete examples.

\section{ACFT-GSUGRA correspondence}

In this section we provide concrete examples of asymmetric Gepner models and make an educated proposal to which $\mathcal{N}=2$ GSUGRA these classical $\mathcal{N}=1$ Minkowski-type string vacua might correspond to. Of course, since the Gepner model is expected to lie deep inside the Kähler moduli space and, in the ACFT, the backreaction from all $\alpha^{\prime}$-corrections has been taken into account, we cannot prove our conjecture but can only collect a number of indications for its correctness.

At the two derivative level the effective action is given by $\mathcal{N}=2$ GSUGRA theory reviewed in the previous section. Let us recall the main features of partial supersymmetry breaking Minkowski vacua. These should be considered as necessary conditions that an $\mathcal{N}=1$ ACFT has to satisfy for admitting an interpretation as a fully adjusted minimum of an associated $\mathcal{N}=2$ GSUGRA theory.

1. As we have seen, the dilaton and a four-dimensional NS-NS pseudo-scalar $\varphi$ remain massless.

2. The constraint on the number of possible gaugings provided the bound

$$
h^{2,1}-h^{1,1}-\Delta \leq N_{V} \leq h^{2,1}-1 .
$$

Since without gaugings/fluxes there are no charged fields under the abelian R-R gauge symmetries, there seems to be no other mechanism to make the U(1)s massive. Thus, we expect this bound to strongly hold also in the ACFTs. 
3. Counting the number of massless axionic chiral multiplets led to the two constraints

$$
\begin{gathered}
N_{V}-N_{\text {ax }} \leq h^{2,1}-h^{1,1}-\Delta, \\
N_{V}-2 N_{\text {ax }} \geq h^{2,1}-2 h^{1,1}-\Delta .
\end{gathered}
$$

This was derived from the tree-level flux induced mass term for the axions and was argued to hold even if higher derivative corrections were taken into account in GSUGRA. However, there exist a possible loop-hole: actually, the axionic Gepner models feature no mass hierarchy between flux induced moduli masses and the KK-scale. Therefore, axionic masses might be generated by including KK modes. This would imply a smaller number of massless axions relaxing the bound in the first relation in (4.2).

4. The number of massless scalars $N_{0}$ is the less constrained one, but at least it should satisfy

$$
N_{0} \leq h^{2,1}+h^{1,1}
$$

Let us mention again that partial $\mathcal{N}=1$ Minkowski-type breaking requires both magnetic and non-geometric gaugings/fluxes. Therefore, if our conjecture is correct, we have identified asymmetric exactly solvable classical string vacua containing in particular nongeometric fluxes.

\subsection{Procedure}

Let us describe how we proceed to identify a candidate GSUGRA model for an ACFT. We start with a usual Gepner model with levels $\left(k_{1}, \ldots, k_{5}\right)$ that is known to correspond to a special point of a Calabi-Yau threefold $\mathcal{M}_{\text {Gep }}$. This is usually a hypersurface in a weighted projective space. Then we extend the modular invariant partition function by a simple current that does not commute with the GSO projection $J_{\mathrm{GSO}}$ and sometimes also not with all of the additional simple currents $J_{i}$. This gives the ACFT featuring a number of massless vectors, R-R axions and scalars $\left(N_{V}, N_{\mathrm{ax}} ; N_{0}\right)$ with $N_{V}>N_{\mathrm{ax}}$. The question now is whether one can find an $\mathcal{N}=2$ SUGRA defined on a Calabi-Yau manifold $\mathcal{M}_{\text {ACFT }}$, whose gauging admits an $\mathcal{N}=1$ Minkowski minimum that is related to the ACFT model.

In order to identify a candidate for $\mathcal{M}_{\mathrm{ACFT}}$, we take a closer look at the massless vectors and try to understand the combinatorics of these states. From this analysis we extract an idea which weights the coordinates and the constraints of $\mathcal{M}_{\text {ACFT }}$ presumably have. The difficulty is that one does not expect a one-to-one correspondence between the massless vectors $N_{V}$ in the ACFT and the massless vectors in the ungauged compactification on $\mathcal{M}_{\mathrm{ACFT}}$ leading to possible ambiguities. This is simply because due to additional gauging some of the vectors of the ungauged theory become massive. Once we isolated a candidate, we check whether the four conditions 1.-4. above are satisfied, i.e. in particular whether the relations (4.1), (4.2) and (4.3) hold. In order to avoid confusion, let us stress that one gets $\mathcal{M}_{\mathrm{ACFT}} \neq \mathcal{M}_{\mathrm{Gep}}$. That additional simple currents usually change the underlying 
manifold can also be seen when using the D-type modular invariant by adding the simple current (2.24). Our procedure can be summarized as

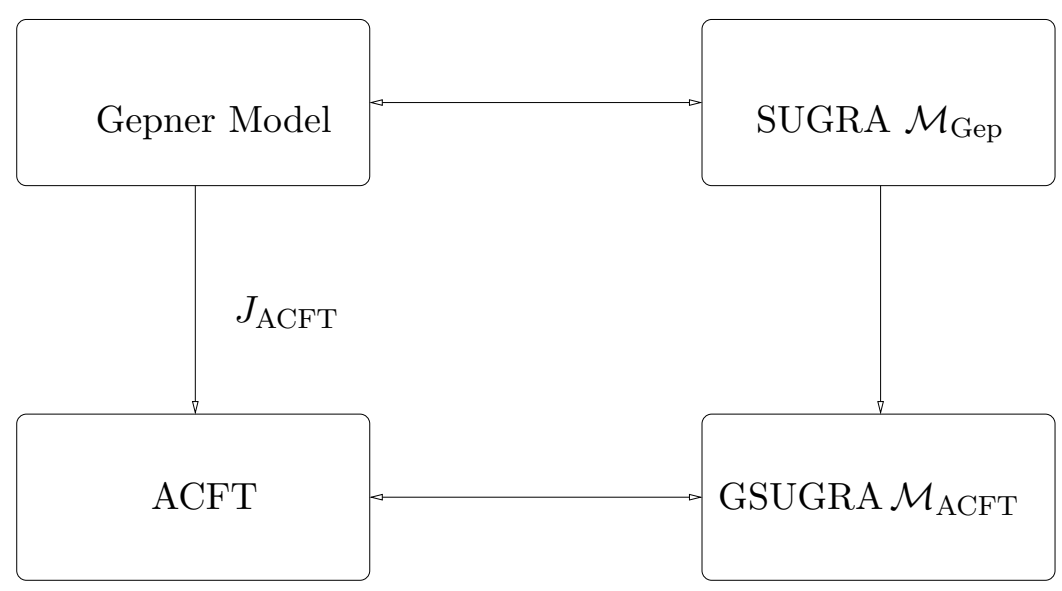

Now let us discuss a couple of examples that will clarify the just described procedure.

\subsection{Odd level ACFT models}

In this section we consider a special class of simple current extended Gepner models $\left(k_{1}, \ldots, k_{5}\right)$. We require that at least one of the levels, say $k_{1}$, is odd so that one has the simple current

$$
J_{\mathrm{ACFT}}=\left(\begin{array}{lll}
0 & k_{1}+2 & 1
\end{array}\right)\left(\begin{array}{lll}
0 & 0 & 0
\end{array}\right)^{4}(c) .
$$

Note that this simple current mixes the R and NS sector between the different minimal models and is non-local with respect to the $J_{i}$ in $(2.23) .{ }^{3}$ Note that these are essentially the simple currents also considered in the $(0,2)$ heterotic Gepner models discussed in [5-7]. Unlike there, here we have the central charge $c=(12,12)$ and therefore rather a type II string theory.

As the most simple example we first discuss the Gepner model with levels $(3,3,3,3,3)$ extended by the simple current

$$
J_{\mathrm{ACFT}}=\left(\begin{array}{lll}
0 & 5 & 1
\end{array}\right)\left(\begin{array}{lll}
0 & 0 & 0
\end{array}\right)^{4}(c) .
$$

This breaks the left-moving supersymmetry and we obtain an $\mathcal{N}=1$ model with massless modes

$$
1 \times(\phi, \varphi)+\left(N_{V}, N_{\mathrm{ax}} ; N_{0}\right)=(80,0 ; 74) .
$$

To get an idea what this model might correspond to, we consider the massless vectors in more detail. First, we note that the pure Gepner model describes a special point in the

\footnotetext{
${ }^{3}$ Notice that this mixing does not necessarily break the left-moving $N=1$ world-sheet supersymmetry. For instance when replacing the $c$ by an $s$ in $J_{\mathrm{ACFT}}$ one finds models with $N=2$ target space SUSY which requires $N=(2,2)$ worldsheet supersymmetry. We think that what is happening here is the following: on each $N=2$ tensor factor one has a spectral flow operator characterized by a parameter $\eta_{i}$. The simple current $J_{\mathrm{ACFT}}$ (or with $c$ and $s$ exchanged) act as a spectral flow operator with $\vec{\eta}=\left(\left(k_{1}+2\right) / 2,0,0,0,0 ; \pm 1 / 2\right)$. If $G^{ \pm}=\sum_{i} 1 \otimes \ldots \otimes G_{i}^{ \pm} \otimes \ldots \otimes 1$ are the world-sheet supercurrents in the former Gepner model, then $G_{\mathrm{ACFT}}^{ \pm}=U_{\vec{\eta}} G^{ \pm} U_{\vec{\eta}}^{\dagger}$ become the left-moving world-sheet supercurrents in the ACFT model. Since it is generated by a spectral flow, one has a full "twisted" $N=2$ super Virasoro algebra.
} 


\begin{tabular}{|c|c|c|}
\hline state & polynom. rep. & deg. \\
\hline$\left(\begin{array}{llll}0 & 1 & 1\end{array}\right)\left(\begin{array}{llll}3 & 4 & 1\end{array}\right)\left(\begin{array}{llll}2 & 3 & 1\end{array}\right)\left(\begin{array}{lll}0 & 1 & 1\end{array}\right)^{2}(s)$ & $x_{i}^{3} x_{j}^{2}$ & 12 \\
\hline$\left(\begin{array}{llll}0 & 1 & 1\end{array}\right)\left(\begin{array}{llll}3 & 4 & 1\end{array}\right)\left(\begin{array}{llll}1 & 2 & 1\end{array}\right)^{2}\left(\begin{array}{llll}0 & 1 & 1\end{array}\right)(s)$ & $x_{i}^{3} x_{j} x_{k}$ & 12 \\
\hline$\left(\begin{array}{lll}0 & 1 & 1\end{array}\right)\left(\begin{array}{lll}2 & 3 & 1\end{array}\right)^{2}\left(\begin{array}{lll}1 & 2 & 1\end{array}\right)\left(\begin{array}{lll}0 & 1 & 1\end{array}\right)(s)$ & $x_{i}^{2} x_{j}^{2} x_{k}$ & 12 \\
\hline$\left(\begin{array}{llll}0 & 1 & 1\end{array}\right)\left(\begin{array}{llll}2 & 3 & 1\end{array}\right)\left(\begin{array}{llll}1 & 2 & 1\end{array}\right)^{3}(s)$ & $x_{i}^{2} x_{j} x_{k} x_{l}$ & 4 \\
\hline \multirow{5}{*}{ 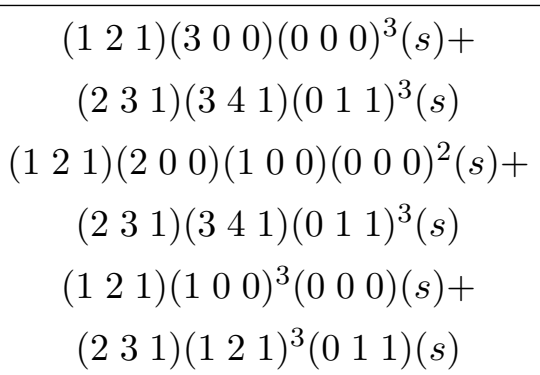 } & $x_{i}^{3} y_{m}$ & $2 \times 4=8$ \\
\hline & & \\
\hline & $x_{i}^{2} x_{j} y_{m}$ & $2 \times 12=24$ \\
\hline & & \\
\hline & $x_{i} x_{j} x_{k} y_{m}$ & $2 \times 4=8$ \\
\hline
\end{tabular}

Table 1. Combinatorics of the $N_{V}=80$ massless vectors.

moduli space of the quintic $\mathcal{M}_{\text {Gep }}=\mathbb{P}_{1,1,1,1,1}[5]^{(101,1)}$. Since the simple current $J_{\mathrm{ACFT}}$ only acts on the first factor, we expect that the other four coordinates $x_{i}$ will still be present. The $N_{V}=80$ massless modes are listed in table 1 . Note that in order the get the combinatorics right, besides the four coordinates of weight one, $\left\{x_{2}, x_{3}, x_{4}, x_{5}\right\}$ we had to introduce two coordinates $\left\{y_{0}, y_{1}\right\}$ of weight two. Thus, all these 80 modes are given by the monomials of order 5 divided by an ideal

$$
\mathcal{P}_{5}\left(x_{i}, y_{j}\right) / \mathcal{I}\left(x_{i}^{4}, y_{j}^{2}, y_{0} y_{1}\right) .
$$

This observation motivates the following proposal for the underlying (fluxed) Calabi-Yau threefold

$$
\mathcal{M}_{\mathrm{ACFT}}=\mathbb{P}_{1,1,1,1,2,2}[53],
$$

i.e. a complete intersection in a weighted projective space. The degree three constraint has been introduced to make it a Calabi-Yau three-fold. This of course introduces more monomials than visible in the ACFT, but recall that due to the gauging we cannot expect a one-to-one correspondence. We rather have to satisfy the bounds (4.1), (4.2) and (4.3).

The ambient space contains a $\mathbb{Z}_{2}$ singularity along the curve $\mathcal{C}_{2}=\mathbb{P}^{1}$ that needs to be resolved. This we do following the method described in appendix A. Using the intersection form on the ambient space $\mathcal{A}$

$$
I_{\mathcal{A}}=\frac{1}{4} \eta^{5}
$$

one computes

$$
\chi_{F}\left(\mathcal{M}_{\mathrm{ACFT}}\right)=-\int_{\mathcal{A}} c_{3}\left(T_{\mathcal{M}}\right) 15 \eta^{2}=-165 .
$$

Thus, the Euler characteristic of the resolved CICY becomes

$$
\chi\left(\mathcal{M}_{\mathrm{ACFT}}\right)=\chi_{F}\left(\mathcal{M}_{\mathrm{ACFT}}\right)-\frac{1}{2} \chi\left(\mathcal{C}_{2}\right)+2 \chi\left(\mathcal{C}_{2}\right)=-162 .
$$


The resolution of the $\mathbb{Z}_{2}$ singularity introduces a single additional Kähler modulus so that the Hodge numbers of the CICY are $\left(h^{2,1}, h^{1,1}\right)=(83,2)$. This agrees with the result of the toric computation as listed in [43] (see also [44, 45]). Though we will discuss it in more detail in section 4.5, let us note that all four necessary conditions 1.-4. are indeed satisfied.

This example can be generalized to an arbitrary Gepner model with say the first level being an odd number $\left(2 l-1, k_{2}, k_{3}, k_{4}, k_{5}\right)$. This Gepner model corresponds to the Fermattype constraint

$$
x_{1}^{2 l+1}+x_{2}^{k_{2}+2}+x_{3}^{k_{3}+2}+x_{4}^{k_{4}+2}+x_{5}^{k_{5}+2}=0
$$

in

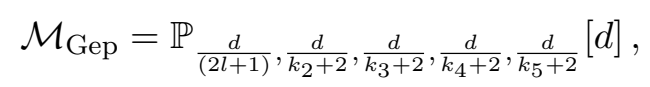

with $d=\operatorname{lcm}\left\{2 l+1, k_{2}+2, k_{3}+2, k_{4}+2, k_{5}+2\right\}$. Extension by the simple current (4.4) leads to an asymmetric Gepner model, for which similar to $[6,7]$ the vectors are given by the combinatorics of polynomials of degree $d$ in the four coordinates $\left(x_{2}, x_{3}, x_{4}, x_{5}\right)$ of weights $w_{i}=d /\left(k_{i}+2\right)$ and two new coordinates $\left(y_{0}, y_{1}\right)$ of weights $w_{0}=2 d /(2 l+1)$ and $w_{1}=l d /(2 l+1)$. Thus, we conjecture that this ACFT corresponds to a Minkowski vacuum of the $\mathcal{N}=2$ GSUGRA on the CICY

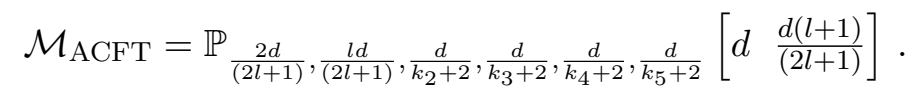

If we have only four tensor factors with say the last level being in addition even, $k_{4}=2 k$, then one can also choose for that factor the D-type modular invariant by adding the simple current (2.24). In this case the Gepner model corresponds to the constraint

$$
x_{1}^{2 l+1}+x_{2}^{k_{2}+2}+x_{3}^{k_{3}+2}+x_{4}^{k+1}+x_{4} x_{5}^{2}=0
$$

in

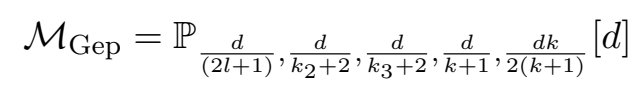

with $d=\operatorname{lcm}\left\{2 l+1, k_{2}+2, k_{3}+2, k+1\right\}$. For this class, the asymmetric Gepner model should correspond to a Minkowski vacuum of the $\mathcal{N}=2$ GSUGRA on the CICY

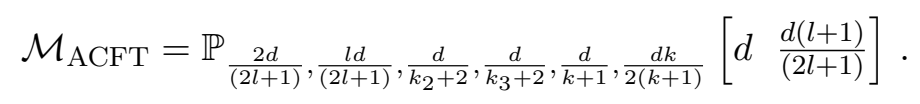

In table 2 we compare the massless spectrum of some of these ACFTs with the Hodge numbers of the CICYs, at least for those cases where the CICY appeared in the list [43]. In section 4.5 we will compare the massless spectrum of these asymmetric Gepner models with the expectation from GSUGRA on the proposed Calabi-Yau three-folds.

Let us emphasize that on the CFT side we have a plethora of consistent models, but most of them cannot be directly related to a GSUGRA theory on a large volume CICY. In fact, most of the spaces (4.14) and (4.17) do not appear in the list of [43] so presumably do not yield transversally intersecting CICYs. 


\begin{tabular}{|c|c|c|}
\hline Gepner & $\left(N_{V}, N_{\mathrm{ax}}, N_{0}\right)$ & CICY \\
\hline$(33333)$ & $(80,0,74)$ & $\mathbb{P}_{1,1,1,1,2,2}[44]_{(83,2)}$ \\
$\left(55512_{D}\right)$ & $(86,2,80)$ & $\mathbb{P}_{1,1,1,2,3,3}[74]_{(89,3)}$ \\
$\left(55512_{A}\right)$ & $(86,2,80)$ & $\mathbb{P}_{1,2,2,4,6,7}[148]_{(88,4)}$ \\
$(77711)$ & $(74,2,70)$ & $\mathbb{P}_{1,1,2,3,3,4}[95]_{(75,6)}$ \\
\hline
\end{tabular}

Table 2. Examples: CFT-GSUGRA correspondence.

\subsection{Level six ACFT model}

In this subsection we consider the Gepner model with levels $\left(6_{A}, 6_{A}, 6_{A}, 6_{D}\right)$ extended by the simple current

$$
J_{\mathrm{ACFT}}=\left(\begin{array}{llll}
0 & 4 & 0
\end{array}\right)\left(\begin{array}{lll}
0 & 0 & 0
\end{array}\right)^{3}(v) .
$$

This simple current is non-local w.r.t. to the simple current $J_{\mathrm{GSO}}$ in (2.23), but being a pure NS-state $J_{\mathrm{ACFT}}$ is still local w.r.t. to all $J_{i}$. As a consequence the left-moving $N=1$ world-sheet supersymmetry is still manifest and one can find the usual $N=1$ supercurrents at the first massive level in the vacuum orbit. We obtain a model with $\mathcal{N}=1$ target-space supersymmetry with massless modes

$$
\left(N_{V}, N_{\text {ax }} ; N_{0}\right)=(60,4 ; 64) .
$$

To get an idea what this model might correspond to, we consider the massless vectors in more detail. First, we note that the pure Gepner model describes a special point in the moduli space of the CY $\mathcal{M}_{\mathrm{Gep}}=\mathbb{P}_{1,1,1,2,3}[8]^{(106,2)}$. Since the simple current $J_{\mathrm{ACFT}}$ only acts on the first factor, we expect that the four coordinates $x_{1}, x_{2}, v, w$ of weights $(1,1,2,3)$ will still be present. The $N_{V}=60$ massless modes are listed in table 3 . Note that all these ACFT states have a twofold degeneracy which we can capture by introducing a factor $s_{\alpha}$ with $\alpha=0,1$ into the corresponding monomials. Moreover, to get the combinatorics right, we introduced the new coordinate $z$ of weight four. Therefore, all these 60 modes are given by the monomials of bi-order $[7,1]$ divided by an ideal

$$
\mathcal{P}_{[7,1]}\left(x_{i[1,0]}, v_{[2,0]}, w_{[3,0]}, z_{[4,0]} ; s_{\alpha[0,1]}\right) / \mathcal{I}\left(x_{i}^{7}, a v^{3}+b w^{2}, v w, s_{1} s_{2}\right)
$$

with $i, \alpha=0,1$. This observation motivates the following proposal for the underlying (fluxed) Calabi-Yau threefold

$$
\mathcal{M}_{\mathrm{ACFT}}=\begin{aligned}
& \mathbb{P}_{1,1,2,3,4} \\
& \mathbb{P}_{1,1}
\end{aligned}\left[\begin{array}{ll}
7 & 4 \\
1 & 1
\end{array}\right],
$$

i.e. a complete intersection in a product of weighted projective spaces.

Of course the first factor $\mathbb{P}_{1,1,2,3,4}$ features $\mathbb{Z}_{2}, \mathbb{Z}_{3}$ and $\mathbb{Z}_{4}$ singularities. Let us discuss their contribution to the Euler characteristic in more detail. First, there exists a single 


\begin{tabular}{|c|c|c|}
\hline State & polynom. rep. & deg. \\
\hline 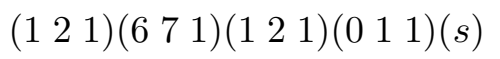 & $s_{\alpha} x_{i}^{6} x_{j}$ & $2 \times 2=4$ \\
\hline$\left(\begin{array}{lll}1 & 2 & 1\end{array}\right)\left(\begin{array}{lll}5 & 6 & 1\end{array}\right)\left(\begin{array}{llll}2 & 3 & 1\end{array}\right)\left(\begin{array}{lll}0 & 1 & 1\end{array}\right)(s)$ & $s_{\alpha} x_{i}^{5} x_{j}^{2}$ & $2 \times 2=4$ \\
\hline$(121)(451)(341)(011)(s)$ & $s_{\alpha} x_{i}^{4} x_{j}^{3}$ & $2 \times 2=4$ \\
\hline$\left(\begin{array}{lll}1 & 2 & 1\end{array}\right)(341)(011)(011)(s)$ & $s_{\alpha} x_{i}^{3} z$ & $2 \times 2=4$ \\
\hline$\left(\begin{array}{llll}1 & 2 & 1\end{array}\right)\left(\begin{array}{llll}2 & 3 & 1\end{array}\right)\left(\begin{array}{llll}1 & 2 & 1\end{array}\right)\left(\begin{array}{lll}0 & 1 & 1\end{array}\right)(s)$ & $s_{\alpha} x_{i}^{2} x_{j} z$ & $2 \times 2=4$ \\
\hline 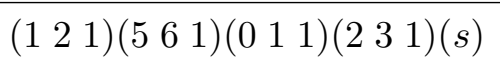 & $s_{\alpha} x_{i}^{5} v$ & $2 \times 2=4$ \\
\hline$(121)(451)(121)(231)(s)$ & $s_{\alpha} x_{i}^{4} x_{j} v$ & $2 \times 2=4$ \\
\hline 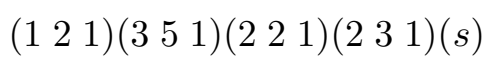 & $s_{\alpha} x_{i}^{3} x_{j}^{2} v$ & $2 \times 2=4$ \\
\hline$\left(\begin{array}{llll}1 & 2 & 1\end{array}\right)\left(\begin{array}{llll}1 & 2 & 1\end{array}\right)\left(\begin{array}{llll}0 & 1 & 1\end{array}\right)\left(\begin{array}{lll}2 & 3 & 1\end{array}\right)(s)$ & $s_{\alpha} x_{i} v z$ & $2 \times 2=4$ \\
\hline$\left(\begin{array}{lll}1 & 2 & 1\end{array}\right)\left(\begin{array}{lll}4 & 5 & 1\end{array}\right)\left(\begin{array}{llll}0 & 1 & 1\end{array}\right)\left(\begin{array}{lll}3 & 4 & 1\end{array}\right)(s)$ & $s_{\alpha} x_{i}^{4} w$ & $2 \times 2=4$ \\
\hline$\left(\begin{array}{lll}1 & 2 & 1\end{array}\right)(3 \quad 41)(121)(341)(s)$ & $s_{\alpha} x_{i}^{3} x_{j} w$ & $2 \times 2=4$ \\
\hline 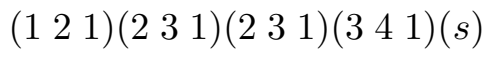 & $s_{\alpha} x_{1}^{2} x_{2}^{2} w$ & $2 \times 1=2$ \\
\hline$\left(\begin{array}{llll}1 & 2 & 1\end{array}\right)\left(\begin{array}{llll}0 & 1 & 1\end{array}\right)\left(\begin{array}{llll}0 & 1 & 1\end{array}\right)\left(\begin{array}{lll}3 & 4 & 1\end{array}\right)(s)$ & $s_{\alpha} w z$ & $2 \times 1=2$ \\
\hline$\left(\begin{array}{llll}1 & 2 & 1\end{array}\right)\left(\begin{array}{llll}3 & 4 & 1\end{array}\right)\left(\begin{array}{lll}0 & 1 & 1\end{array}\right)\left(\begin{array}{lll}4 & 5 & 1\end{array}\right)(s)$ & $s_{\alpha} x_{i}^{3} v^{2}$ & $2 \times 2=4$ \\
\hline$(121)(241)(121)(451)(s)$ & $s_{\alpha} x_{i}^{2} x_{j} v^{2}$ & $2 \times 2=4$ \\
\hline 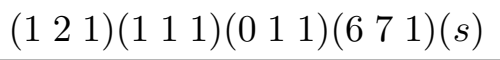 & $s_{\alpha} x_{i} w^{2}\left(\sim v^{3}\right)$ & $2 \times 2=4$ \\
\hline
\end{tabular}

Table 3. Combinatorics of the $N_{V}=60$ massless vectors

point $\mathcal{P}_{4}$ over which one has a $\mathbb{Z}_{4}$ singularity. Second, one has a $\mathbb{Z}_{3}$ singularity over the curve $\mathcal{C}_{3}=\mathbb{P}^{1}$. Finally, the CICY has a $\mathbb{Z}_{2}$ singularity over a curve

$$
\mathcal{C}_{2}=\mathbb{P}_{1,2}\left[\begin{array}{l}
2 \\
\mathbb{P}_{1,1}
\end{array}\right],
$$

containing the former $\mathbb{Z}_{4}$ singularity. Using the methods described in appendix A, we obtain for the Euler characteristics of the singular loci

$$
\begin{gathered}
\chi\left(\mathcal{C}_{3}\right)=2, \quad \chi\left(\mathcal{P}_{4}\right)=1, \\
\chi\left(\mathcal{C}_{2} / \mathcal{P}_{4}\right)=\chi_{F}\left(\mathcal{C}_{2}\right)-\frac{1}{2} \chi\left(\mathcal{P}_{4}\right)=1 .
\end{gathered}
$$

Using the intersection form on the ambient space $\mathcal{A}$

$$
I_{\mathcal{A}}=\frac{1}{24} \eta_{1}^{4} \eta_{2}
$$

one computes

$$
\chi_{F}\left(\mathcal{M}_{\mathrm{ACFT}}\right)=-\int_{\mathcal{A}} c_{3}\left(T_{\mathcal{M}}\right)\left(7 \eta_{1}+\eta_{2}\right)\left(4 \eta_{1}+\eta_{2}\right)=-\frac{1471}{12} .
$$




\begin{tabular}{|c|c|}
\hline polynom. rep. & deg. \\
\hline$p_{11}(x)$ & 18 \\
$p_{10}(x)$ & 19 \\
$p_{7}(x)$ & $10+2$ \\
$p_{4}(x)$ & 6 \\
$p_{3}(x)$ & 3 \\
\hline
\end{tabular}

Table 4. Combinatorics of the $N_{V}=59$ massless vectors in terms of polynomials $p_{n}\left(x_{2}, x_{3}, x_{4}\right) / \mathcal{I}\left(x_{2}^{11}, x_{3}^{5}, x_{4}^{5}\right)$.

Thus, the Euler characteristic of the resolved CICY becomes

$$
\begin{aligned}
\chi\left(\mathcal{M}_{\mathrm{ACFT}}\right)= & \chi_{F}\left(\mathcal{M}_{\mathrm{ACFT}}\right)-\frac{1}{2} \chi\left(\mathcal{C}_{2} / \mathcal{P}_{4}\right)-\frac{1}{3} \chi\left(\mathcal{C}_{3}\right)-\frac{1}{4} \chi\left(\mathcal{P}_{4}\right) \\
& +2 \chi\left(\mathcal{C}_{2} / \mathcal{P}_{4}\right)+3 \chi\left(\mathcal{C}_{3}\right)+4 \chi\left(\mathcal{P}_{4}\right) \\
= & -112 .
\end{aligned}
$$

Moreover, besides the two toric Kähler classes, the resolution introduces the following number of extra Kähler classes

$$
\mathbb{Z}_{2}: h^{1,1}=1, \quad \mathbb{Z}_{3}: h^{1,1}=2, \quad \mathbb{Z}_{4}: h^{1,1}=1,
$$

so that the Hodge numbers of the resolved CICY are $\left(h^{2,1}, h^{1,1}\right)=(62,6)$.

\subsection{Level ten ACFT model}

Finally let us briefly discuss the Gepner model $(10,10,4,4)$ with only $A$-type modular invariants that corresponds to the Calabi-Yau $\mathcal{M}_{\text {Gep }}=\mathbb{P}_{1,1,2,2,6}[12]$. We extend the partition function again by the simple current

$$
J_{\mathrm{ACFT}}=\left(\begin{array}{lll}
0 & 4 & 0
\end{array}\right)\left(\begin{array}{lll}
0 & 0 & 0
\end{array}\right)^{3}(v),
$$

yielding an $\mathcal{N}=1$ Minkowski vacuum with massless modes

$$
\left(N_{V}, N_{\mathrm{ax}} ; N_{0}\right)=(59,5 ; 68)
$$

In terms of the three unaffected coordinates $\left\{x_{2}, x_{3}, x_{4}\right\}$ of weights $\{1,2,2\}$ all the massless states follow the combinatorics shown in table 4 .

Furthermore one sees that the states fall into a twisted and an untwisted sector. Arranging the degrees according to these sectors into two sets $\{11,7,3\}$ and $\{10,7,4\}$, one might be tempted to introduce two extra coordinates of weights three and four. Taking also the maximally appearing degrees 11 and 10 into account, we conjecture that the underlying Calabi-Yau threefold could be

$$
\mathcal{M}_{\mathrm{ACFT}}=\mathbb{P}_{1,2,2,3,4,9}\left[\begin{array}{ll}
11 & 10
\end{array}\right] .
$$


Of course some of the possible monomials appearing in $\mathcal{M}_{\mathrm{ACFT}}$ are missing on the ACFT side, but that is expected due to the gauging. Employing the methods from appendix A we can derive the Hodge numbers $\left(h^{2,1}, h^{1,1}\right)=(66,8)$. At least these numbers lie in the right ballpark. However, this example shows that it is not straightforward to identify a candidate GSUGRA model. It always involves a bit of guess-work and intuition.

\subsection{Check of GSUGRA constraints}

In this section we check whether the massless spectra for the asymmetric Gepner models are consistent with the necessary constraints 1.-4. from the effective GSUGRA analysis. To explicitly check whether there exist concrete choices for the fluxes that admit these GSUGRA vacua is a difficult though interesting question.

First of all, all presented examples of ACFTs do contain a universal massless chiral multiplet from the vacuum orbit. This is the candidate for hosting the dilaton and a NS-NS pseudo-scalar. In GSUGRA we have seen that the latter could be the $\tilde{\phi}$-field itself or a linear combination of the many NS-NS pseudo-scalars residing in the complex structure and complexified Kähler moduli. Second, for GSUGRA we have derived the bounds (4.1), and (4.2) on the number of massless vector and chiral RR-axion multiplets. The mild bound (4.3) on the number of scalars is satisfied for all examples. In table 5 we compare the ACFT data with the GSUGRA bounds. One realizes that the bounds are fairly strong, not leaving much freedom for the number of massless vectors and R-R axions. For instance for the asymmetric $(3)^{5}$ Gepner model the GSUGRA constraints only admit the six possible spectra given by $\left(N_{V}, N_{\text {ax }}\right) \in\{(80,0),(80,1),(81,0),(81,1),(82,1),(82,2)\}$.

For the number of vector multiplets the bounds are always satisfied. Recall that this is certainly the mostly protected sector. As indicated in table 5, there exists one case where the GSUGRA conditions for the RR-axions are not satisfied. For the asymmetric $\left(1^{2} 7^{3}\right)$ Gepner model, the GSUGRA predicts too many massless R-R axions. Therefore, this models seems to need some dynamics that is not captured by $\mathcal{N}=2$ GSUGRA.

Apart from that we consider the correspondence between the massless spectra of $\mathcal{N}=1$ asymmetric Gepner models and partially broken $\mathcal{N}=2$ GSUGRA very encouraging and would like to conjecture that the ACFTs do really describe the fully backreacted solutions, that are indicated by Minkowski vacua of an GSUGRA approximation.

Again, we are not claiming that the latter gives a completely established Wilsonian

effective description. Instead, as argued in [40-42], large parts of the full dynamics are dictated by the tree level potential and might be protected enough such that there are indeed solutions that survive in the full string theory after adjusting themselves.

\section{Conclusions}

In this paper we have collected some evidence that a certain class of asymmetric Gepner models can be identified with fully backreacted vacua, that are indicated by partially broken $\mathcal{N}=1$ Minkowski minima of corresponding $\mathcal{N}=2$ GSUGRA theories. Our work goes beyond the former attempts $[8,35,36]$ in that we took a closer look at the massless states in the ACFTs and came up with concrete proposals for the CICYs underlying the GSUGRA 


\begin{tabular}{|c|c|c|c|}
\hline Gepner & $\left(N_{V}, N_{\mathrm{ax}}, N_{0}\right)$ & $\left(h^{2,1}, h^{1,1}\right)$ & constraints \\
\hline$(33333)$ & $(80,0,74)$ & $(83,2)$ & $N_{V}-N_{\mathrm{ax}} \leq 81-\Delta$ \\
& & & $N_{V}-2 N_{\mathrm{ax}} \geq 79-\Delta$ \\
& & & \\
& & & \\
& & $(89,3)$ & $N_{V}-N_{\mathrm{ax}} \leq 86-\Delta$ \\
& & & $N_{V}-2 N_{\mathrm{ax}} \geq 83-\Delta$ \\
$\left.-55512_{D}\right)$ & $(86,2,80)$ & $86-\Delta \leq N_{V} \leq 88$ \\
\hline$\left(55512_{A}\right)$ & $(86,2,80)$ & $(88,4)$ & $N_{V}-N_{\mathrm{ax}} \leq 84-\Delta$ \\
& & & $N_{V}-2 N_{\mathrm{ax}} \geq 80-\Delta$ \\
& & & $84-\Delta \leq N_{V} \leq 87$ \\
\hline$(77711)$ & $(74,2,70)$ & $(75,6)$ & $N_{V}-N_{\mathrm{ax}} \leq 69-\Delta$ \\
& & & $N_{V}-2 N_{\mathrm{ax}} \geq 63-\Delta$ \\
& & & $69-\Delta \leq N_{V} \leq 74$ \\
\hline$\left(6666_{D}\right)$ & $(60,4,64)$ & $(62,6)$ & $N_{V}-N_{\mathrm{ax}} \leq 56-\Delta$ \\
& & & $N_{V}-2 N_{\mathrm{ax}} \geq 50-\Delta$ \\
& & & $56-\Delta \leq N_{V} \leq 61$ \\
\hline$(101044)$ & $(59,5,68)$ & $(66,8)$ & $N_{V}-N_{\mathrm{ax}} \leq 58-\Delta$ \\
& & & $N_{V}-2 N_{\mathrm{ax}} \geq 50-\Delta$ \\
& & $58-\Delta \leq N_{V} \leq 65$ \\
\hline
\end{tabular}

Table 5. Check: ACFT-GSUGRA correspondence. The underlined condition is not satisfied while the dashed conditions are satisfied if only one of the RR-axions $\zeta_{1,2}$ remains massless after being fixed by (3.19).

theories. It is almost inevitable that there exists some ambiguity in the choice of the underlying CY manifold, as due to the flux some of the axions and scalars have already become massive. Since for our examples there seems to be only few moduli missing, the number of fluxes turned on is expected to be rather small. We derived a number of constraints for the massless spectra for $\mathcal{N}=1$ minima of GSUGRA that were almost all satisfied by the candidates for the ACFT-GSUGRA correspondence. Moreover, this picture fits perfectly with the expectation that non-geometric fluxes are related to asymmetric CFTs.

Clearly, we were just collecting arguments but could not give a complete proof of our conjecture. It would be desirable to be more concrete about precisely which fluxes have been turned on, but that requires the knowledge of the period matrices of complex structure and Kähler moduli in the vicinity of a small radius Gepner point. For the CICYs appearing in our list, this is not known. 
The class of ACFT that we were considering is huge and only very few models could be identified with large volume geometries. In general we expect that these ACFTs only exist in the stringy regime not admitting any geometric interpretation.

\section{Acknowledgments}

We are grateful to S. Greiner, T.W. Grimm, D. Junghans and E. Malek for helpful discussions.

\section{A Hodge numbers for CICY}

In this appendix we review the employed technique to determine the Hodge numbers of CICYs in products of weighted projective spaces $[46,47]$. The latter are generically singular so that one has to resolve them.

Say we want to compute the Hodge numbers of $\mathbb{P}_{w_{1}, \ldots, w_{6}}\left[d_{1} d_{2}\right]$. The intersection form in the in general singular ambient space is

$$
I_{\mathcal{A}}=\frac{1}{\prod_{i} w_{i}} \eta^{5}
$$

Whenever some of the coordinates have a common divisor $N$, one has a $\mathbb{Z}_{N}$ singularity over the locus where the remaining coordinates vanish. As long as the intersection of these loci with the two hypersurface constraints leads to singular points and curves $\mathcal{D}$, the CICY can be resolved in a Ricci-flat manner. The Euler characteristic of the resolution can be computed via

$$
\chi(M)=\chi_{F}\left(M_{\text {sing }}\right)-\frac{1}{N} \chi(\mathcal{D})+N \chi(\mathcal{D})
$$

where the rational number $\chi_{F}\left(M_{\text {sing }}\right)$ can be computed via

$$
\chi_{F}\left(M_{\text {sing }}\right)=-\int_{\mathcal{A}} c_{3}\left(T_{M}\right)\left(d_{1} d_{2} \eta^{2}\right) .
$$

The third Chern class can be read off from the total Chern class

$$
c\left(T_{M}\right)=\left.\frac{\left(1+d_{1} \eta\right)\left(1+d_{2} \eta\right)}{\prod_{i}\left(1+w_{i} \eta\right)}\right|_{\eta^{5}} .
$$

It often happens that the various singularities do intersect. In this case the above formulas have to be iterated such that each singularity is only counted ones. How this works, is demonstrated for the examples explicitly discussed the main text of this paper.

The number of Kähler classes can be computed in the following way. Besides the canonical $(1,1)$ forms inherited from the ambient space, from the resolution of singular curves and points one gets:

- The resolution of a singular curve of order $N$ introduces $(N-1)$ additional $(1,1)$ forms. 
- The resolution of a singular point of order $N$ introduces $\frac{1}{2}(N-1)$ additional $(1,1)$ forms.

- If on top of a singular curve of order $N$ there are singular points of order $N \cdot M$ for each such point one deducts $\frac{1}{2}(N-1)(1,1)$-forms.

Open Access. This article is distributed under the terms of the Creative Commons Attribution License (CC-BY 4.0), which permits any use, distribution and reproduction in any medium, provided the original author(s) and source are credited.

\section{References}

[1] D. Gepner, Space-Time Supersymmetry in Compactified String Theory and Superconformal Models, Nucl. Phys. B 296 (1988) 757 [INSPIRE].

[2] D. Gepner, Exactly Solvable String Compactifications on Manifolds of SU(N) Holonomy, Phys. Lett. B 199 (1987) 380 [InSPIRE].

[3] A.N. Schellekens and S. Yankielowicz, Extended Chiral Algebras and Modular Invariant Partition Functions, Nucl. Phys. B 327 (1989) 673 [InSPIRE].

[4] A.N. Schellekens and S. Yankielowicz, Modular Invariants From Simple Currents: An Explicit Proof, Phys. Lett. B 227 (1989) 387 [INSPIRE].

[5] R. Blumenhagen and A. Wisskirchen, Exactly solvable (0,2) supersymmetric string vacua with GUT gauge groups, Nucl. Phys. B 454 (1995) 561 [hep-th/9506104] [INSPIRE].

[6] R. Blumenhagen, R. Schimmrigk and A. Wisskirchen, The $(0,2)$ exactly solvable structure of chiral rings, Landau-Ginzburg theories and Calabi-Yau manifolds, Nucl. Phys. B 461 (1996) 460 [hep-th/9510055] [INSPIRE].

[7] R. Blumenhagen, R. Schimmrigk and A. Wisskirchen, $(0,2)$ mirror symmetry, Nucl. Phys. B 486 (1997) 598 [hep-th/9609167] [INSPIRE].

[8] A.N. Schellekens and S. Yankielowicz, New Modular Invariants for $\mathcal{N}=2$ Tensor Products and Four-dimensional Strings, Nucl. Phys. B 330 (1990) 103 [InSPIRE].

[9] B. Gato-Rivera and A.N. Schellekens, Asymmetric Gepner Models: Revisited, Nucl. Phys. B 841 (2010) 100 [arXiv: 1003.6075] [INSPIRE].

[10] B. Gato-Rivera and A.N. Schellekens, Asymmetric Gepner Models II. Heterotic Weight Lifting, Nucl. Phys. B 846 (2011) 429 [arXiv: 1009.1320] [INSPIRE].

[11] M. Graña, R. Minasian, M. Petrini and A. Tomasiello, Generalized structures of $\mathcal{N}=1$ vacua, JHEP 11 (2005) 020 [hep-th/0505212] [INSPIRE].

[12] M. Graña, J. Louis and D. Waldram, Hitchin functionals in $\mathcal{N}=2$ supergravity, JHEP 01 (2006) 008 [hep-th/0505264] [INSPIRE].

[13] I. Benmachiche and T.W. Grimm, Generalized $\mathcal{N}=1$ orientifold compactifications and the Hitchin functionals, Nucl. Phys. B 748 (2006) 200 [hep-th/0602241] [INSPIRE].

[14] M. Graña, J. Louis and D. Waldram, $\mathrm{SU}(3) \times \mathrm{SU}(3)$ compactification and mirror duals of magnetic fluxes, JHEP 04 (2007) 101 [hep-th/0612237] [INSPIRE].

[15] D. Cassani and A. Bilal, Effective actions and $\mathcal{N}=1$ vacuum conditions from $\mathrm{SU}(3) \times \mathrm{SU}(3)$ compactifications, JHEP 09 (2007) 076 [arXiv:0707.3125] [INSPIRE]. 
[16] G. Aldazabal, D. Marques and C. Núñez, Double Field Theory: A Pedagogical Review, Class. Quant. Grav. 30 (2013) 163001 [arXiv:1305.1907] [INSPIRE].

[17] D.S. Berman and D.C. Thompson, Duality Symmetric String and M-theory, Phys. Rept. 566 (2014) 1 [arXiv: 1306.2643] [INSPIRE].

[18] O. Hohm, D. Lüst and B. Zwiebach, The Spacetime of Double Field Theory: Review, Remarks and Outlook, Fortsch. Phys. 61 (2013) 926 [arXiv:1309.2977] [InSPIRE].

[19] R. Blumenhagen, A. Font and E. Plauschinn, Relating double field theory to the scalar potential of $\mathcal{N}=2$ gauged supergravity, JHEP 12 (2015) 122 [arXiv:1507.08059] [INSPIRE].

[20] J. Louis and A. Micu, Type 2 theories compactified on Calabi-Yau threefolds in the presence of background fluxes, Nucl. Phys. B 635 (2002) 395 [hep-th/0202168] [INSPIRE].

[21] G. Dall'Agata, String compactifications with fluxes, Class. Quant. Grav. 21 (2004) S1479 [INSPIRE].

[22] R. D'Auria, S. Ferrara, M. Trigiante and S. Vaula, Gauging the Heisenberg algebra of special quaternionic manifolds, Phys. Lett. B 610 (2005) 147 [hep-th/0410290] [INSPIRE].

[23] R. D'Auria, S. Ferrara, M. Trigiante and S. Vaula, Scalar potential for the gauged Heisenberg algebra and a non-polynomial antisymmetric tensor theory, Phys. Lett. B 610 (2005) 270 [hep-th/0412063] [INSPIRE].

[24] R. D'Auria, S. Ferrara and M. Trigiante, On the supergravity formulation of mirror symmetry in generalized Calabi-Yau manifolds, Nucl. Phys. B 780 (2007) 28 [hep-th/0701247] [INSPIRE].

[25] S. Ferrara, L. Girardello and M. Porrati, Minimal Higgs branch for the breaking of half of the supersymmetries in $\mathcal{N}=2$ supergravity, Phys. Lett. B 366 (1996) 155 [hep-th/9510074] [INSPIRE].

[26] D. Cassani, S. Ferrara, A. Marrani, J.F. Morales and H. Samtleben, A special road to AdS vacua, JHEP 02 (2010) 027 [arXiv:0911.2708] [INSPIRE].

[27] J. Louis, P. Smyth and H. Triendl, Spontaneous $\mathcal{N}=2$ to $\mathcal{N}=1$ Supersymmetry Breaking in Supergravity and Type II String Theory, JHEP 02 (2010) 103 [arXiv:0911.5077] [InSPIRE].

[28] J. Louis, P. Smyth and H. Triendl, The $\mathcal{N}=1$ Low-Energy Effective Action of Spontaneously Broken $\mathcal{N}=2$ Supergravities, JHEP 10 (2010) 017 [arXiv:1008.1214] [INSPIRE].

[29] T. Hansen and J. Louis, Examples of $\mathcal{N}=2$ to $\mathcal{N}=1$ supersymmetry breaking, JHEP 11 (2013) 075 [arXiv: 1306.5994] [INSPIRE].

[30] R. Blumenhagen et al., A Flux-Scaling Scenario for High-Scale Moduli Stabilization in String Theory, Nucl. Phys. B 897 (2015) 500 [arXiv:1503.07634] [INSPIRE].

[31] A. Dabholkar and C. Hull, Duality twists, orbifolds and fluxes, JHEP 09 (2003) 054 [hep-th/0210209] [INSPIRE].

[32] A. Flournoy and B. Williams, Nongeometry, duality twists and the worldsheet, JHEP 01 (2006) 166 [hep-th/0511126] [INSPIRE].

[33] C. Condeescu, I. Florakis and D. Lüst, Asymmetric Orbifolds, Non-Geometric Fluxes and Non-Commutativity in Closed String Theory, JHEP 04 (2012) 121 [arXiv:1202.6366] [INSPIRE]. 
[34] C. Condeescu, I. Florakis, C. Kounnas and D. Lüst, Gauged supergravities and non-geometric Q/R-fluxes from asymmetric orbifold CFT's, JHEP 10 (2013) 057 [arXiv: 1307.0999] [INSPIRE].

[35] D. Israël and V. Thiéry, Asymmetric Gepner models in type-II, JHEP 02 (2014) 011 [arXiv: 1310.4116] [INSPIRE].

[36] D. Israël, Nongeometric Calabi-Yau compactifications and fractional mirror symmetry, Phys. Rev. D 91 (2015) 066005 [arXiv: 1503.01552] [INSPIRE].

[37] R. Blumenhagen and E. Plauschinn, Introduction to conformal field theory, Lect. Notes Phys. 779 (2009) 1.

[38] D. Cassani, Reducing democratic type-II supergravity on $\mathrm{SU}(3) \times \mathrm{SU}(3)$ structures, JHEP 06 (2008) 027 [arXiv: 0804.0595] [INSPIRE].

[39] L. Andrianopoli, R. D'Auria, S. Ferrara and M.A. Lledó, $\mathcal{N}=2$ superHiggs, $\mathcal{N}=1$ Poincaré vacua and quaternionic geometry, JHEP 01 (2003) 045 [hep-th/0212236] [INSPIRE].

[40] N. Kaloper and L. Sorbo, A Natural Framework for Chaotic Inflation, Phys. Rev. Lett. 102 (2009) 121301 [arXiv:0811.1989] [INSPIRE].

[41] N. Kaloper, A. Lawrence and L. Sorbo, An Ignoble Approach to Large Field Inflation, JCAP 03 (2011) 023 [arXiv: 1101.0026] [INSPIRE].

[42] S. Bielleman, L.E. Ibáñez and I. Valenzuela, Minkowski 3-forms, Flux String Vacua, Axion Stability and Naturalness, JHEP 12 (2015) 119 [arXiv:1507.06793] [INSPIRE].

[43] A. Klemm, M. Kreuzer, E. Riegler and E. Scheidegger, Topological string amplitudes, complete intersection Calabi-Yau spaces and threshold corrections, JHEP 05 (2005) 023 [hep-th/0410018] [INSPIRE].

[44] M. Kreuzer, E. Riegler and D.A. Sahakyan, Toric complete intersections and weighted projective space, J. Geom. Phys. 46 (2003) 159 [math/0103214] [INSPIRE].

[45] A. Klemm, M. Kreuzer, E. Riegler and E. Scheidegger, Topological string amplitudes, complete intersection Calabi-Yau spaces and threshold corrections, JHEP 05 (2005) 023 [hep-th/0410018] [INSPIRE].

[46] J. Fuchs, A. Klemm, C. Scheich and M.G. Schmidt, Gepner Models With Arbitrary Affine Invariants and the Associated Calabi-Yau Spaces, Phys. Lett. B 232 (1989) 317 [InSPIRE].

[47] J. Fuchs, A. Klemm, C. Scheich and M.G. Schmidt, Spectra and Symmetries of Gepner Models Compared to Calabi-Yau Compactifications, Annals Phys. 204 (1990) 1 [INSPIRE]. 